\title{
Short-Duration Swimming Exercise after Myocardial Infarction Attenuates Cardiac Dysfunction and Regulates Mitochondrial Quality Control in Aged Mice
}

\author{
Dajun Zhao, ${ }^{1}$ Yang Sun, ${ }^{2}$ Yanzhen Tan, ${ }^{1}$ Zhengbin Zhang, ${ }^{2}$ Zuoxu Hou, ${ }^{3}$ Chao Gao, ${ }^{4}$ \\ Pan Feng, ${ }^{1}$ Xing Zhang, ${ }^{3}$ Wei Yi $\left(\mathbb{D},{ }^{1}\right.$ and Feng Gao ${ }^{3}{ }^{3}$ \\ ${ }^{1}$ Department of Cardiovascular Surgery, Xijing Hospital, The Fourth Military Medical University, 127 Changle West Road, \\ Xi'an 710032, China \\ ${ }^{2}$ Department of Geriatric, Xijing Hospital, The Fourth Military Medical University, 127 Changle West Road, Xi'an 710032, China \\ ${ }^{3}$ Department of Aerospace Medicine, The Fourth Military Medical University, 169 Changle West Road, Xi'an 710032, China \\ ${ }^{4}$ Department of Cardiology, Xijing Hospital, The Fourth Military Medical University, 127 Changle West Road, Xi'an 710032, China
}

Correspondence should be addressed to Wei Yi; yiwei@fmmu.edu.cn and Feng Gao; fgao@fmmu.edu.cn

Received 12 September 2017; Revised 21 December 2017; Accepted 24 January 2018; Published 11 April 2018

Academic Editor: Zsolt Radák

Copyright (c) 2018 Dajun Zhao et al. This is an open access article distributed under the Creative Commons Attribution License, which permits unrestricted use, distribution, and reproduction in any medium, provided the original work is properly cited.

Background. Exercise benefits to cardiac rehabilitation (CR) following stable myocardial infarction (MI). The suitable exercise duration for aged patients with coronary heart disease (CHD) remains controversial, and the underlying molecular mechanism is still unclear. Methods and Results. 18-Month-old mice after stable MI were randomly submitted to different durations of exercise, including 15 and 60 min swimming training (ST) once per day, five times a week for 8 weeks. Compared to sedentary mice, $15 \mathrm{~min}$ ST, rather than $60 \mathrm{~min}$ ST, significantly augmented left ventricular function, increased survival rate, and suppressed myocardial fibrosis and apoptosis. $15 \mathrm{~min}$ ST improved mitochondrial morphology via regulating mitochondrial fission-fusion signaling. $15 \mathrm{~min}$ ST regulated mitophagy signaling via inhibiting LC3-II and P62 levels and increasing PINK/Parkin expression. 15 min ST also inhibited ROS production and enhanced antioxidant SOD2 activity. Notably, 15 min ST significantly increased sirtuin (SIRT) 3 level (2.7-fold) in vivo while the inhibition of SIRT3 exacerbated senescent H9c2 cellular LDH release and ROS production under hypoxia. In addition, SIRT3 silencing impairs mitochondrial dynamics and mitophagy in senescent cardiomyocytes against simulated ischemia (SI) injury. Conclusion. Collectively, our study demonstrated for the first time that sustained short-duration exercise, rather than long-duration exercise, attenuates cardiac dysfunction after MI in aged mice. It is likely that the positive regulation induced by a short-duration ST regimen on the elevated SIRT3 protein level improved mitochondrial quality control and decreased apoptosis and fibrosis contributed to the observed more resistant phenotype.

\section{Introduction}

Physical exercise is an effective therapy for patients with stable coronary heart disease (CHD). Exercise-based cardiac rehabilitation (CR) reduces all-cause mortality, declines rehospitalization, and improves health-related quality of life following myocardial infarction (MI) [1-3]. However, aging greatly influences exercise parameters and cardiac performances [4]. Elderly patients with CHD are more prone to deconditioning, frailty, and disability with intrinsic muscle weakness, joint instability, and metabolic risks, leading to the difficult implementation of exercise. Moreover, the association between the duration of exercise and the cardioprotective effects remains controversial $[5,6]$. Whether aged heart benefits distinctly from different durations of exercise as rehabilitative modalities is not yet understood.

Among the complex determinants of aging, mitochondrial dysfunction is identified as one of the major causes [7]. The maintenance of an adequate pool of functional mitochondria is crucial for cardiomyocyte homeostasis [8]. 
Mitochondrial dysfunction triggers mitochondrial quality control to remove damaged components, recover mitochondrial morphology, and repair cardiomyocyte function responding to stresses [9]. Mitochondrial quality control consists of a series of mechanisms, including reactive oxygen species (ROS) scavenging, mitochondrial dynamics, and mitophagy. Studies have revealed that exercise improves mitochondrial quality both in healthy [10] and in metabolic and neurodegenerative disorders as well as in aging $[11,12]$.

Sirtuin, also known as Sir2 proteins, is a family of nicotinamide adenine dinucleotide-dependent deacetylases. SIRT3 is one of the sirtuin family members that localizes in mitochondria [13]. It inhibits ROS production and maintains mitochondrial functions $[14,15]$. Studies have demonstrated that SIRT3 protects against acute myocardial ischemia injury [16] while SIRT3 deficiency significantly inhibits angiogenesis and cardiac functional recovery following MI [17]. Notably, SIRT3 expression is proved to be modified by exercise training both in humans and murines [18], suggesting that SIRT3 might be crucial to exercise's rehabilitative effects against MI injury consequences.

The aims of this study were (1) to determine whether exercise rehabilitates cardiac dysfunction after $\mathrm{MI}$ in aged mice; (2) to investigate the suitable exercise duration for CR; (3) to elucidate whether mitochondrial quality control is associated with rehabilitative effects of exercise after $\mathrm{MI}$; and (4) to examine the potential role for SIRT3 in the process.

\section{Materials and Methods}

All animal experiments were approved by the Fourth Military Medical University Committee on Animal Care. C57BL/6J male mice were provided by the Experimental Animal Center of the Fourth Military Medical University (Xi'an, Shaanxi, China). Mice were maintained in $12 \mathrm{~h}: 12 \mathrm{~h}$ artificial light-dark cycles, with lights on at 6:00 AM, and were housed individually in the lab's animal room. H9c2 cardiac cell lines were purchased from the American Type Culture Collection (ATCC ${ }^{\circledR} \mathrm{CRL}-1446^{\mathrm{TM}}$ ).

2.1. Mouse Model of MI. 18-Month-old male C57BL/6J mice were anesthetized with $2 \%$ isoflurane. Myocardial infarction (MI) was produced via the ligation of left coronary artery as previously described [19]. Sham-operated control mice (21 mice) underwent the same procedures except that the suture placed under the left coronary artery was not tied.

2.2. Exercise Protocol. Four months after stable MI, 68 alive mice were randomly divided into three groups: (1) sedentary (MI-sedentary, 22 mice); (2) short-duration swimming training (ST) $\left(\mathrm{MI}+15^{\prime} \mathrm{ST}, 23\right.$ mice); and (3) long-duration ST (MI +60' ST, 23 mice). The ST protocol was adapted from previously published procedures [20, 21]. Briefly, mice in groups of three to five animals were trained 5 days per week for 8 weeks in the $60 \mathrm{~cm} \times 90 \mathrm{~cm}$ buckets filled with $\approx 20 \mathrm{~cm}$ depth of water at $33-35^{\circ} \mathrm{C}$. The ST lasted $15 \mathrm{~min}$ on the first day. Then the exercise duration was maintained at $15 \mathrm{~min} /$ day in the $\mathrm{MI}+15^{\prime} \mathrm{ST}$ group, or progressively increased to $60 \mathrm{~min} /$ day in the $\mathrm{MI}+60$ ' ST group during the first week. All training sessions took place during the morning hours (9:00 AM-11:00 AM). Mouse physical parameters and the survival rate were assessed before and during the ST period. Meanwhile, sedentary animals were housed individually in cages as a control.

2.3. Echocardiography. Mice (8-9 per group) were submitted to transthoracic echocardiography (VisualSonics Vevo 2100 Imaging System) to evaluate the cardiac structure and function before and during the ST period. M-mode tracings were taken and echocardiogram analysis was performed.

2.4. Masson Trichrome Staining. Mouse hearts (6-8 mice per group) were perfused with ice-cold phosphate buffered saline (PBS) and were fixed with $4 \%$ paraformaldehyde, embedded in paraffin, and coronally sectioned $(3-6 \mu \mathrm{m}$ thick). Sections were stained using a Masson trichrome stain assay (Sigma-Aldrich, HT15-1KT) according to the manufacturer's instructions.

2.5. TUNEL Staining. Mouse hearts (6-8 mice per group) were fixed with $4 \%$ paraformaldehyde. Sections were stained with TUNEL staining assay (Roche Diagnostics Corporation, 11684817910) and $\alpha$-actin antibody (Sigma-Aldrich, A7811) to detect myocardial apoptotic cells according to the manufacturer's instructions.

2.6. Detection of ROS Production. Mouse hearts (6-8 mice per group) were embedded in optimal cutting temperature compound (OCT) and were frozen immediately after euthanasia. Frozen sections (7-10 $\mu \mathrm{m}$ thick) were cut by a cryomicrotome (Model CM3050S, Leica Microsystems) and incubated with dihydroethidium (DHE, $2 \mathrm{mM}$; Molecular Probes, D1168) for 1 hour at room temperature. Slides were examined by a laser scanning confocal microscope (FluoView ${ }^{\mathrm{TM}}$ FV1000, Olympus). The numbers of DHE-positive nuclei and the total nuclei were counted (100 fields per group).

Cardiomyocyte mitochondrial superoxide generation was assessed using the MitoSOX Red dye (Molecular Probes, Invitrogen, M36008). Living cells were incubated with $5 \mathrm{mM}$ MitoSOX Red at $37^{\circ} \mathrm{C}$ for $10 \mathrm{~min}$ and were examined by the laser scanning confocal microscope.

2.7. Transmission Electron Microscopy. Left ventricular tissues (6 mice per group) were fixed with $2.5 \%$ glutaraldehyde in $0.1 \mathrm{M}$ sodium phosphate $(\mathrm{pH} 7.4)$ overnight at $4^{\circ} \mathrm{C}$. After postfixation in $1 \% \mathrm{OsO}_{4}$, samples were dehydrated through graded alcohols and embedded in Epon Araldite. Ultrathin sections $(50 \mathrm{~nm})$ were cut by an ultramicrotome (Ultracut E, Leica) and stained with uranyl acetate and lead citrate. The specimens were viewed on a Tecnai G2 Spirit electron microscope (FEI Co., Hillsboro, OR). Images were captured at $15,000 \mathrm{x}$ magnification. Mitochondrial shape descriptors (including aspect ratio, circularity, roundness, and solidity) and area measurements were obtained using Image (version 1.42q, National Institutes of Health, Bethesda, MD). A total of 243 mitochondria from the sham group, 257 mitochondria from the MI-sedentary group, 240 mitochondria from the MI+15' ST group, and 251 mitochondria 
from the MI +60' ST group were analyzed from five electron micrographs. The frequency distribution of the mitochondrial area was determined by GraphPad Prism 6 statistic software, yielding frequency histograms.

2.8. Isolation of Mitochondria. Mitochondria were isolated using mitochondria isolation kits (Beyotime Institute of Biotechnology, c3606 for tissue and c3601 for cell) according to the manufacturer's instructions. Briefly, fresh mouse hearts ( 6 to 8 mice per group) were minced in the centrifuge tube. The pellet was homogenized in the trypsin-EDTA $(8 \mu \mathrm{L} / \mathrm{mg})$ solution and centrifuged at $600 \mathrm{~g}$ for $20 \mathrm{~s}$. After washing, the pellet was resuspended with $8 \mu \mathrm{L} / \mathrm{mg}$ mitochondria separation reagent $\mathrm{B}$ combined with PMSF and transferred into a prechilled glass homogenizer. The suspension was homogenized for 20-30 times on the ice and centrifuged at $600 \mathrm{~g}$ for $5 \mathrm{~min}$. Mitochondria were pelleted from the supernatant via centrifugation at $11,000 \mathrm{~g}$ for $10 \mathrm{~min}$. The final pellet was either resuspended in $40 \mu \mathrm{L} / \mathrm{mg}$ mitochondria store liquid for intact function assay or lysed for Western blot analysis.

For cellular mitochondria isolation, H9c2 cardiomyocytes at a density of $5 \times 10^{7}$ cells were harvested and resuspended in a $2.5 \mathrm{~mL}$ mitochondria isolation buffer. The suspension was homogenized for 15-20 times in the glass homogenizer and centrifuged at $600 \mathrm{~g}$ for $10 \mathrm{~min}$. Then, the supernatant was centrifuged at $11,000 \mathrm{~g}$ for $10 \mathrm{~min}$. The final mitochondrial pellet was lysed for Western blot analysis.

2.9. Induction of Cell Senescence and SA- $\beta$-gal Staining. H9c2 cardiomyocytes were cultured in high-glucose Dulbecco's modified Eagle's medium (DMEM) supplemented with $10 \%$ fetal bovine serum (FBS), $100 \mathrm{U} / \mathrm{mL}$ penicillin, and $100 \mathrm{mg} / \mathrm{mL}$ streptomycin at $37^{\circ} \mathrm{C}$ with $5 \% \mathrm{CO}_{2}$. Doxorubicin (DOX, Sigma-Aldrich, D1515) was diluted with 5\% glucose immediately before use. Cultured cells were treated with $\operatorname{DOX}(0.1 \mu \mathrm{M})$ for $24 \mathrm{~h}$ to induce senescence and $5 \%$ glucose was used as a vehicle control. Senescence-associated- $\beta$ galactosidase (SA- $\beta$-gal, Beyotime Institute of Biotechnology, C0602) staining was used to detect cell senescence as previously described [22-24]. The number of SA- $\beta$-galpositive cells was determined by a microscope $(\times 100)$ and expressed as a percentage of all counted cells (Suppl. Figure $1 \mathrm{~A}$ and $\mathrm{B}$ ).

2.10. In Vitro Lentivirus-Mediated SIRT3 Silencing. H9c2 cardiomyocytes were grown in a 6-well plate to approximate $80 \%$ confluence $\left(\approx 5 \times 10^{4}\right.$ cells $)$. Cultured cells were transfected with SIRT3-ShRNA lentivirus $\left(5 \times 10^{8} \mathrm{Tu} / \mathrm{mL}\right.$, Shanghai GenePharma Co. Ltd., Lv3-SIRT3-Rat-597) or a negative control (NC, $1 \times 10^{8} \mathrm{Tu} / \mathrm{mL}$, Shanghai GenePharma Co. Ltd., Lv3NC) at 100 multiply of infection (MOI) in the presence of $5 \mu \mathrm{g} / \mathrm{mL}$ polybrene according to the previously published studies $[25,26]$. Cells infected with GFP-SIRT3 lentivirus were observed under a microscope $(\times 100)$. The silence efficiency was determined by Western blot.

2.11. Simulated Ischemia (SI). Normal culture medium was replaced with Hank's balanced salt solution (HBSS, Gibco, 14025076), and cardiomyocytes were placed in a Napco
8000WJ hypoxia $\left(1 \% \mathrm{O}_{2}, 5 \% \mathrm{CO}_{2}\right.$, and $\left.94 \% \mathrm{~N}_{2}\right)$ incubator (Thermo Scientific) for $12 \mathrm{~h}$ as previously described [27].

2.12. Cell Viability. H9c2 cellular viability was analyzed after SIRT3 silencing followed by hypoxia. CCK-8 assay (SigmaAldrich, 96992) was used according to the manufacturer's instructions. Briefly, cells were incubated with $5 \mathrm{mg} / \mathrm{mL}$ CCK- 8 in a $\mathrm{CO}_{2}$ incubator for $3 \mathrm{~h}$. The medium was aspirated, and the absorbance of each well was measured by the plate reader at a test wavelength of $460 \mathrm{~nm}$ with a reference wavelength of $630 \mathrm{~nm}$. Optical density (OD) was utilized as the indicator of the cell survival rate.

2.13. Western Blot Analysis. Whole heart lysate and mitochondrial lysate were used in the present study. The protein concentrations were determined by a BCA protein assay kit (Thermo Fisher, 23225). Proteins were separated through electrophoresis and transferred to PVDF membranes. The membranes were blocked for 2 hours in 5\% nonfat dry milk and were subsequently incubated overnight at $4^{\circ} \mathrm{C}$ with appropriate primary antibodies against Drp1 (Cell Signaling Technology, number 8570), Fis1 (Enzo Life Sciences, ALX210-1037), Mfn1 (Santa Cruz Biotechnology, sc-166644), Mfn2 (Sigma-Aldrich, M9073), Opa1 (BD Biosciences, 612606), COX IV (Cell Signaling Technology, number 4844), LC3A/B (Cell Signaling Technology, number 2775), P62 (Cell Signaling Technology, number 39749), PINK1 (Cell Signaling Technology, number 6946), Parkin (Cell Signaling Technology, number 4211), SIRT3 (Abgent, number AP6242a), GAPDH (CMCTAG Inc., AT0002), HSP60 (Cell Signaling Technology, number 12165), PGC1- $\alpha$ (Cell Signaling Technology, number 2178), SOD2 (Abcam, ab13533), and Ac-SOD2 (Abcam, ab137037). The membranes were washed by Tris-buffered saline containing $0.1 \%$ Tween 20 (TBST, pH 7.6) and then subsequently probed with appropriate secondary antibodies (Zhongshan Company, ZB-2301, $\mathrm{ZB}-2305$ ) at room temperature for $90 \mathrm{~min}$. The protein bands were detected using a Bio-Rad imaging system (Hercules, CA, USA) and normalized to COX IV or GAPDH.

2.14. Statistical Analysis. All values in the text and figures are presented as the mean \pm standard error of the mean (SEM) of $n$ independent experiments. The data were analyzed using GraphPad Prism 6 statistic software (La Jolla, CA, USA). Data were submitted to $t$-test (two groups) or one-way ANOVA (three or more groups). Data of cellular experiment were determined with two-way ANOVA followed by post hoc tests with Holm adjustment. $P$ values of $<0.05$ (two sided) were considered to be statistically significant.

\section{Results}

3.1. Short-Duration Exercise after MI Attenuates Cardiac Dysfunction and Improves the Survival Rate in Aged Mice. To determine exercise's effects upon post-MI injury in aged mice, mouse physical and cardiovascular parameters were measured before and after 8-week ST. Mouse body weight was decreased after swimming for 8 weeks $(P<$ 0.05 , resp., Figure 1(a)) whereas only $15 \mathrm{~min}$ ST mice manifested a lower ratio of heart weight to body weight 


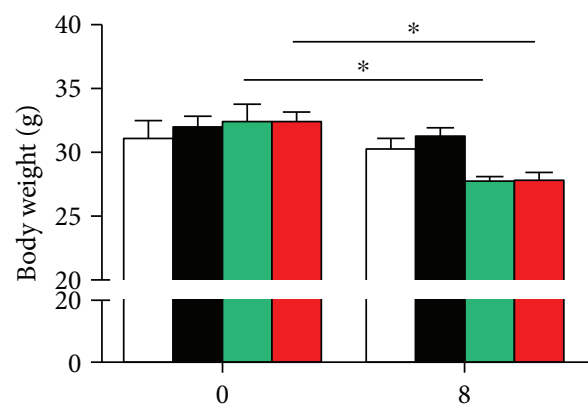

(Week)

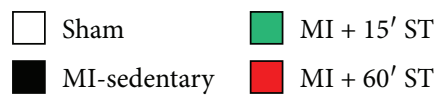

(a)

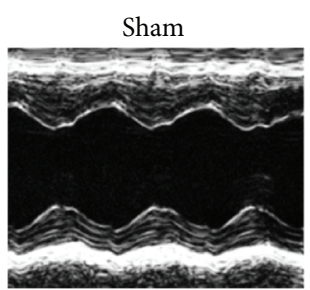

MI-sedentary
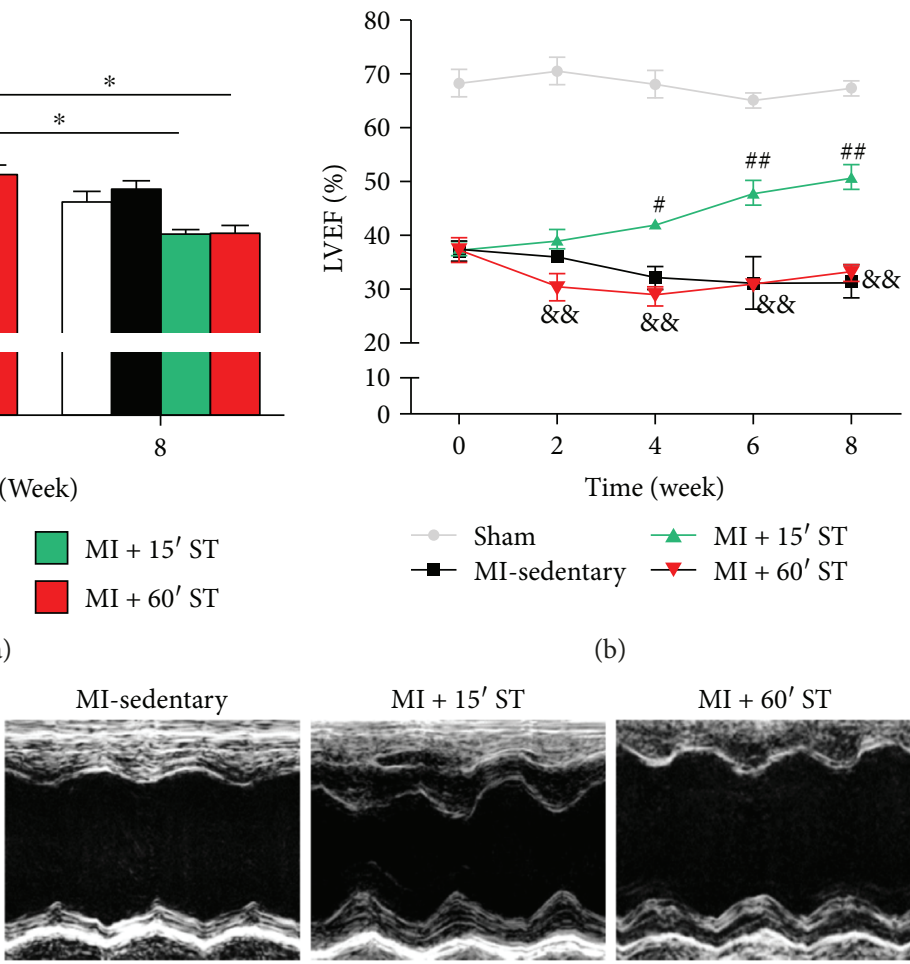

$\rightarrow$ Sham $\rightarrow \mathrm{MI}+15^{\prime} \mathrm{ST}$

$\rightarrow$ MI-sedentary $\rightarrow \mathrm{MI}+60^{\prime} \mathrm{ST}$

(b)

$\mathrm{MI}+15^{\prime} \mathrm{ST}$

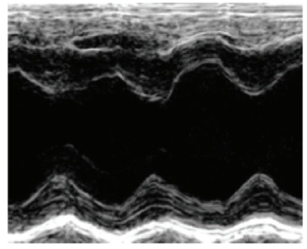

$\mathrm{MI}+60^{\prime} \mathrm{ST}$

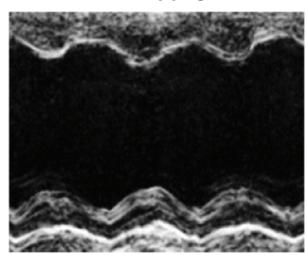

(c)

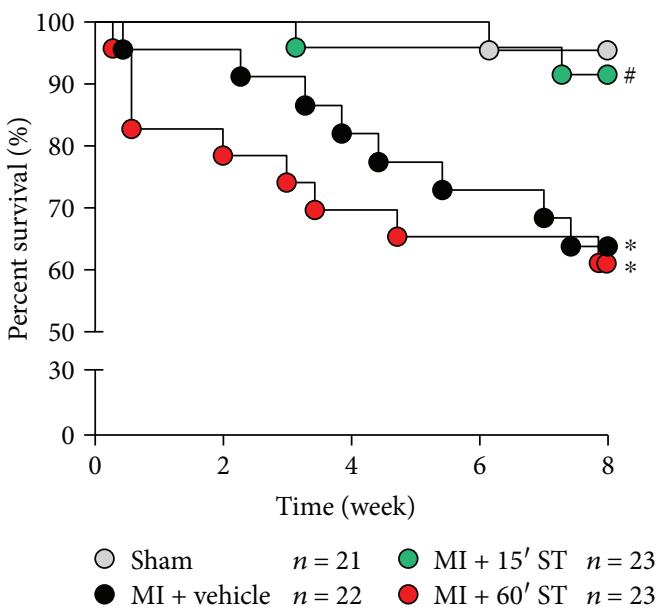

(d)

FIGURE 1: Short-duration exercise after MI promotes left ventricular ejection fraction (LVEF) and reduces mortality in aged mice. (a) Statistics of mouse body weight before and after 8-week swimming training (ST). (b) LVEF analysis. (c) Echocardiography of aged mice after 8-week ST. (d) Survival curves. ${ }^{*} P<0.05$ versus the sham group; ${ }^{\#} P<0.05$, ${ }^{\# \#} P<0.01$ versus the MI-sedentary group; \&\& $P<0.01$ versus the MI $+15^{\prime}$ ST group. $N=21-23$ for survival rate analysis and $N=8-9$ for other assessments.

$(P<0.05$, Table 1). Echocardiographic results (Figures 1(b) and $1(\mathrm{c})$ ) revealed that post-MI mouse left ventricular ejection fraction (LVEF) was augmented after $15 \mathrm{~min}$ ST for 4 weeks $(P<0.01)$ and continued increasing for 8 weeks. Furthermore, $15 \mathrm{~min}$ ST improved the mouse post-MI survival rate $(P<0.05)$ compared to the MI-sedentary group (Figure 1(d)). However, $60 \mathrm{~min}$ ST mice manifested no significant changes in phenotype. Taken together, these data demonstrate that short-duration rather than long-duration exercise protects against post-MI injury in aged mice.
3.2. Short-Duration Exercise after MI Inhibits Cardiac Fibrosis and Apoptosis in Aged Mice. Then, we investigated whether different durations of ST affected cardiac fibrosis and apoptosis, the primary causes of cardiac remodeling and heart failure. $15 \mathrm{~min}$ ST mice manifested a lower myocardial interstitial fibrosis $(P<0.01$, Figures $2(a)$ and $2(\mathrm{~b}))$ and less myocardial apoptosis $(P<0.01$, Figures $2(\mathrm{c})$ and $2(\mathrm{~d}))$ compared to the MI-sedentary group. However, 60 min ST mice performed an increase of myocardial interstitial fibrosis $(P<0.05$, Figures $2(\mathrm{a})$ and $2(\mathrm{~b}))$ with no change 
Table 1: Physical and echocardiographic parameters of aged mice after 8 weeks of swimming training.

\begin{tabular}{|c|c|c|c|c|}
\hline \multirow[b]{2}{*}{ Parameter } & \multicolumn{4}{|c|}{ Mean \pm SEM } \\
\hline & $\begin{array}{l}\text { Sham } \\
(n=8)\end{array}$ & $\begin{array}{l}\text { MI-sedentary } \\
\quad(n=9)\end{array}$ & $\begin{array}{c}\mathrm{MI}+15^{\prime} \mathrm{ST} \\
\quad(n=9)\end{array}$ & $\begin{array}{c}\mathrm{MI}+600^{\prime} \mathrm{ST} \\
(n=8)\end{array}$ \\
\hline \multicolumn{5}{|c|}{ Physical characteristic } \\
\hline $\mathrm{BW}(\mathrm{g})$ & $30.3 \pm 0.73$ & $31.2 \pm 0.71$ & $27.7 \pm 0.35^{*}$ & $27.7 \pm 0.66^{*}$ \\
\hline Heart (mg) & $140 \pm 4.7$ & $200 \pm 4.7^{*}$ & $130 \pm 3.7^{\# \#}$ & $180 \pm 7.8^{*, \& \&}$ \\
\hline $\mathrm{HW} / \mathrm{BW}(\mathrm{mg} / \mathrm{g})$ & $4.6 \pm 0.22$ & $6.4 \pm 0.26^{*}$ & $4.7 \pm 0.13^{\#}$ & $6.6 \pm 0.37^{*, \&}$ \\
\hline Tibial length $(\mathrm{cm})$ & $2.3 \pm 0.04$ & $2.3 \pm 0.04$ & $2.3 \pm 0.04$ & $2.3 \pm 0.03$ \\
\hline $\mathrm{HW} / \mathrm{TL}(\mathrm{mg} / \mathrm{cm})$ & $61.0 \pm 2.44$ & $87.5 \pm 3.24^{*}$ & $57.5 \pm 1.83^{\#}$ & $80.0 \pm 3.40^{*, \&}$ \\
\hline \multicolumn{5}{|l|}{ Echocardiography } \\
\hline LVEF (\%) & $67.8 \pm 2.21$ & $31.3 \pm 2.24^{*}$ & $49.5 \pm 1.82^{*, \# \#}$ & $34.6 \pm 2.14^{*, \& \&}$ \\
\hline LVFS (\%) & $38.3 \pm 0.98$ & $17.5 \pm 1.46^{*}$ & $25.1 \pm 1.59^{*, \#}$ & $19.7 \pm 1.47^{*, \&}$ \\
\hline LVEDD (mm) & $4.0 \pm 0.13$ & $5.2 \pm 0.23^{*}$ & $4.4 \pm 0.09^{\#}$ & $5.5 \pm 0.30^{*, \&}$ \\
\hline $\operatorname{LVESD}(\mathrm{mm})$ & $2.3 \pm 0.18$ & $3.9 \pm 0.16^{*}$ & $2.9 \pm 0.09^{\#}$ & $3.8 \pm 0.28^{*, \&}$ \\
\hline
\end{tabular}

${ }^{*} P<0.05$ versus the sham group; ${ }^{\#} P<0.05$, \#\# $P<0.01$ versus the MI-sedentary group; ${ }^{\&} P<0.05$, \&\& $P<0.01$ versus the MI +15 ' ST group. $N=8-9$. BW, body weight; HW, heart weight; TL, tibial length; LVEF, left ventricular ejection fraction; LVFS, left ventricular fractional shortening; LVEDD, left ventricular end-diastolic diameter; LVESD, left ventricular end-systolic diameter.

of cardiac apoptosis $(P=0.16$, Figures $2(\mathrm{c})$ and $2(\mathrm{~d}))$. In summary, short-duration exercise attenuates postischemic cardiac fibrosis and apoptosis in aged mice.

3.3. Short-Duration Exercise after MI Restores Mitochondrial Morphology and Regulates Mitochondrial Dynamics Signaling in Aged Heart. Mitochondrial impairment directly contributes to aging and age-related diseases; we examined cardiac mitochondrial ultrastructure by transmission electron microscopy (TEM). As shown in Figure 3(a), mitochondria in the MI-sedentary group were disorganized and in large clusters with small, round mitochondria. Mitochondrial shape was distinctly alerted with the declined aspect ratio $(P<0.01)$ and increased circularity $(P<0.05)$ and roundness $(P<0.01)$ compared to that in the sham group (Table 2). Moreover, the frequency distribution for mitochondrial size was highly skewed post-MI (Figure 3(b)). 15 min ST improved mitochondria to be more homogeneous in size (Figures 3(a) and 3(b)).

However, a large number of swelling mitochondria with broken or dismissing ridge were observed in $60 \mathrm{~min}$ ST mouse hearts (Figure 3(a)).

To determine whether mitochondrial dynamics contribute to the impaired mitochondrial morphology, we quantified mitochondrial fission-fusion signaling markers. Aged mice after MI manifested significant increases of fissionrelated protein Drp1 (2.5-fold) and fusion-related protein Mfn1 and Mfn2 levels $(P<0.01$, resp., Figures 3(c)-3(e), $3(\mathrm{~g})$ and $3(\mathrm{~h})$ ), in consistent with the TEM results. $15 \mathrm{~min}$ ST reduced the levels of Mfn1, Mfn2, and Drp1 $(P<0.01$, resp.) while increased Opal expression $(P<0.01)$. Differently, 60 min ST inhibited Mfn1, Mfn2, and Drp1 expression $(P<0.05$, resp.) with no alteration of Opal (Figures 3(c)3(e), 3(g)-3(i)). However, no changes of fission-related Fis1 levels (Figures 3(c) and 3(f)) were detected in each group. These results suggest that short-duration exercise after MI regulates mitochondrial fission-fusion signaling to recover mitochondrial morphology in aged mice.

3.4. Short-Duration Exercise after MI Regulates Mitophagy Signaling in Aged Mice. To evaluate exercise's effect on mitophagy, we quantified the expression of mitophagy signalingrelated markers. The levels of mitochondrial LC3-II were increased in MI-sedentary mice $(P<0.01)$ and $60 \mathrm{~min}$ ST mice $(P<0.01)$ but were declined in $15 \mathrm{~min}$ ST mice $(P<$ $0.01)$ compared to that in the sham group (Figures $4(\mathrm{a})$ and 4(b)). Levels of p62 manifested similar changes on ST (Figures 4(a) and 4(c)). The increases of LC3-II levels might derive from excessive mitophagy or from downstream block of autophagic vacuole processing. To distinguish between these 2 possibilities, we assessed PINK1 and Parkin expression, two proteins involved in mitophagy initiation. We found that MI injury suppressed PINK1 and Parkin expression $(P<0.01$, resp.). Their levels were restored when exposed to $15 \mathrm{~min}$ ST $(P<0.01$, resp.) rather than $60 \mathrm{~min}$ ST (Figures $4(\mathrm{~d})$ and $4(\mathrm{e})$ ). These data suggest that shortduration exercise after MI regulates mitophagy signaling biomarkers in aged heart.

3.5. Short-Duration Exercise after MI Attenuates Oxidative Stress and Increases Mitochondrial SIRT3 Expression in Aged Heart. Furthermore, we tested exercise's effects on oxidative stress. Aged mice after MI manifested a great accumulation of superoxide compared to the sham group (4.6-fold, $P<0.01$ ). 15 min ST distinctly inhibited ROS production for $48.3 \%(P<0.01)$ while $60 \mathrm{~min}$ ST slightly reduced $15.9 \%$ ROS production $(P<0.01)$ (Figures 5(a) and $5(\mathrm{~b}))$. Moreover, $15 \mathrm{~min}$ ST mice manifested higher antioxidant SOD2 activity determined by the decrease of SOD2 acetylation compared to the MI-sedentary group $(P<0.01$, Figure 5(c)). Together, these findings support that short-duration exercise protects against post-MI injury 

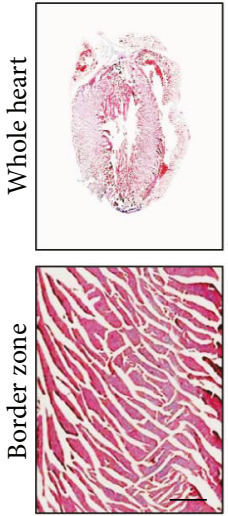

Sham
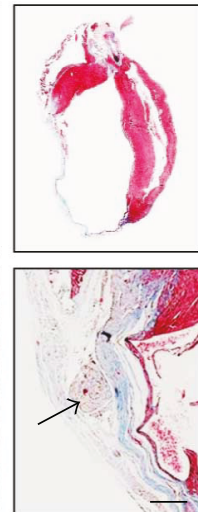

MI-sedentary
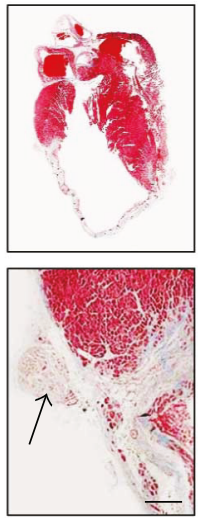

$\mathrm{MI}+15^{\prime} \mathrm{ST}$
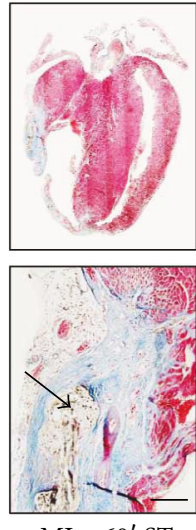

$\mathrm{MI}+60^{\prime} \mathrm{ST}$

(a)
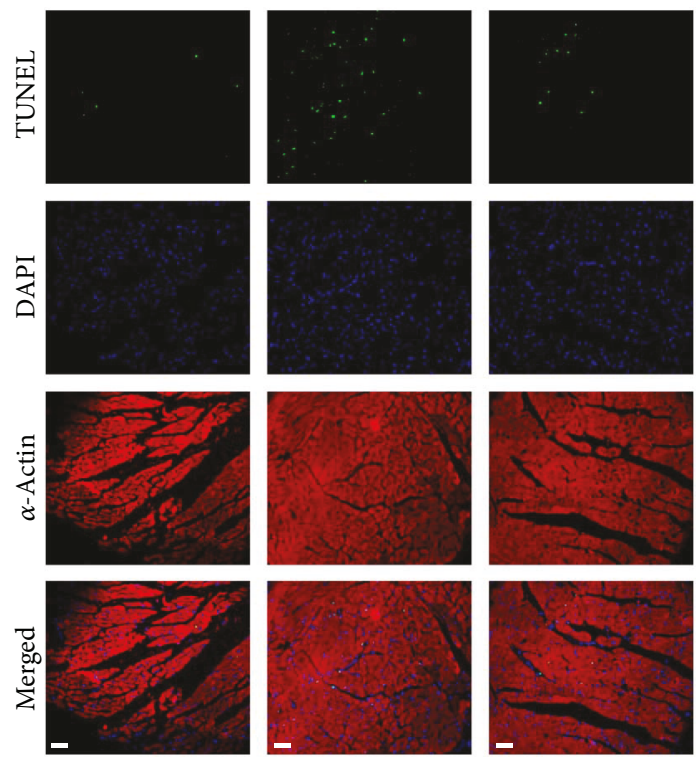

Sham

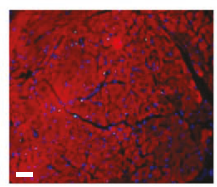

MI-sedentary
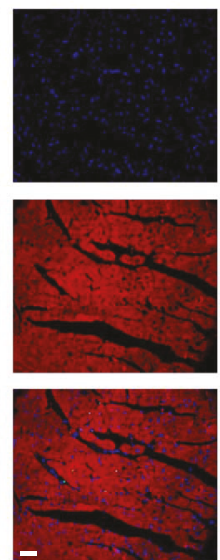

$\mathrm{MI}+15^{\prime} \mathrm{ST}$
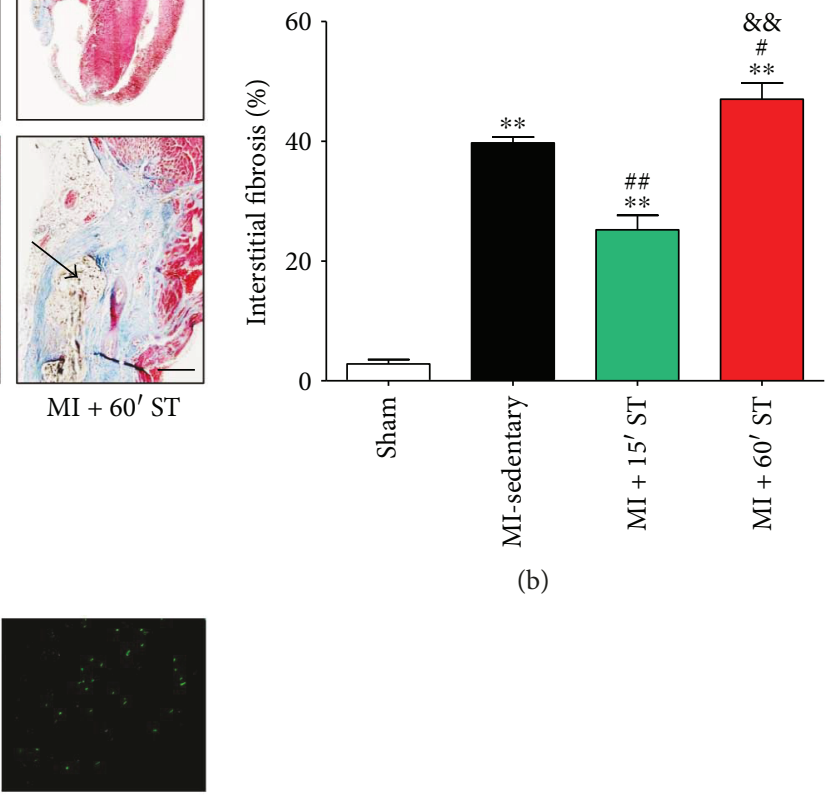

(b)
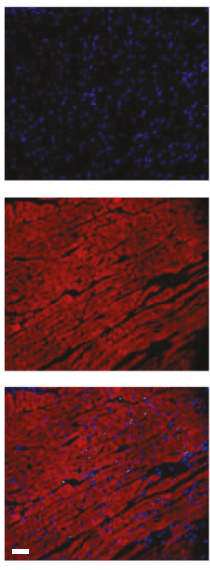

$\mathrm{MI}+60^{\prime} \mathrm{ST}$

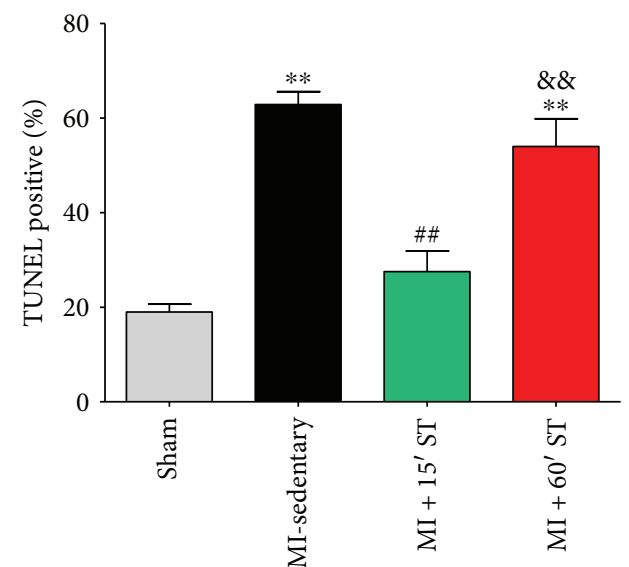

(d)

FIgure 2: Short-duration exercise after MI inhibits cardiac fibrosis and apoptosis in aged mice. (a, b) Myocardial interstitial fibrosis determined by Masson trichrome staining. Black arrows indicate the ligation of left coronary artery. Scale bars, $50 \mu \mathrm{m}$. (c, d) Cardiomyocyte apoptosis determined by TUNEL staining. Scale bars, $30 \mu \mathrm{m} .{ }^{* *} P<0.01$ versus the sham group; ${ }^{\#} P<0.05$, ${ }^{\# \#} P<0.01$ versus the MI-sedentary group; ${ }^{\& \&} P<0.01$ versus the $\mathrm{MI}+15$ ' ST group. $N=6-8$.

via antioxidant actions in aged mice. Notably, mitochondrial SIRT3 expression was increased 2.7 -fold under $15 \mathrm{~min}$ ST $(P<0.01)$, but with no change via $60 \mathrm{~min}$ ST $(P=0.81)$, compared to that in the MI-sedentary group (Figure $5(\mathrm{~d})$ ). It indicates that SIRT3 might play a critical role in shortduration exercise's cardioprotections.

3.6. SIRT3 Deficiency Exacerbates SI-Induced Senescenced Cardiomyocyte Apoptosis and ROS Production In Vitro. To further determine the cardioprotective role of SIRT3 in aging, we used lentivirus to knockdown SIRT3 expression in $\mathrm{H} 9 \mathrm{c} 2$ cardiomyocytes. Compared to negative control (NC), the level of cellular SIRT3 was dramatically decreased by $74.5 \%$ in the SIRT3-ShRNA group $(P<0.01$, Suppl. Figure $1 \mathrm{C}$ and D), confirming the lentivirus silence efficiency. Cell viability was measured via CCK- 8 assay, and the inhibition of SIRT3 expression significantly increased SI-induced cell death $(P<0.01$, Suppl. Figure 1E). To confirm our finding, $\mathrm{LDH}$ release and caspase- 3 activity were performed. In the $\mathrm{SI}+\mathrm{NC}$ group, cellular $\mathrm{LDH}$ release was approximately 

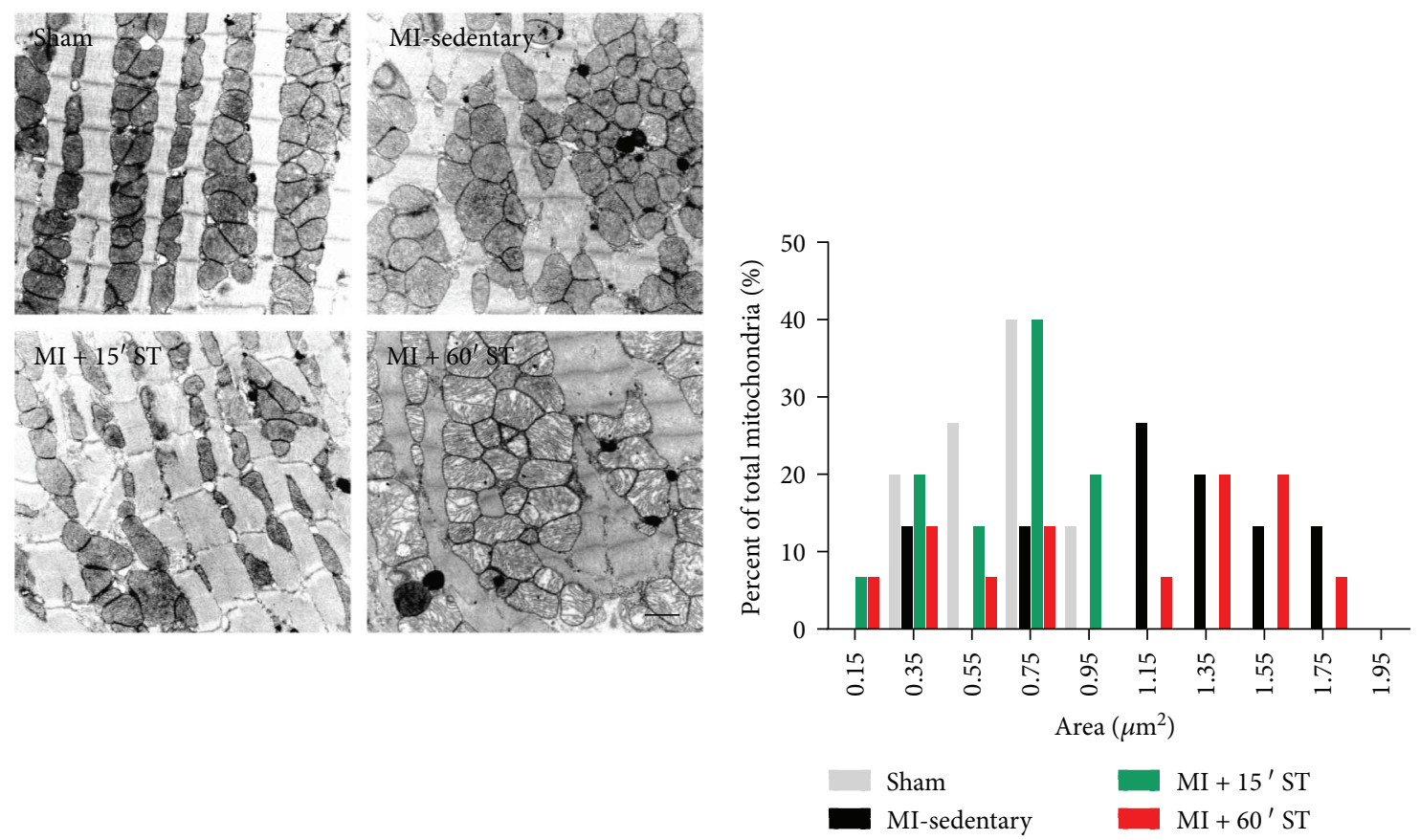

(a)

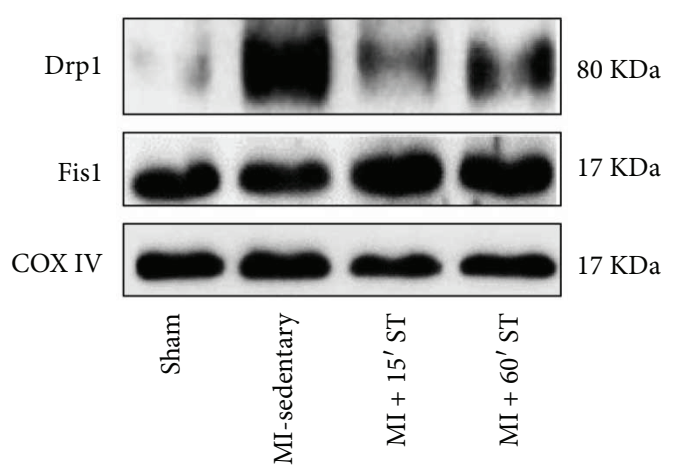

(c)

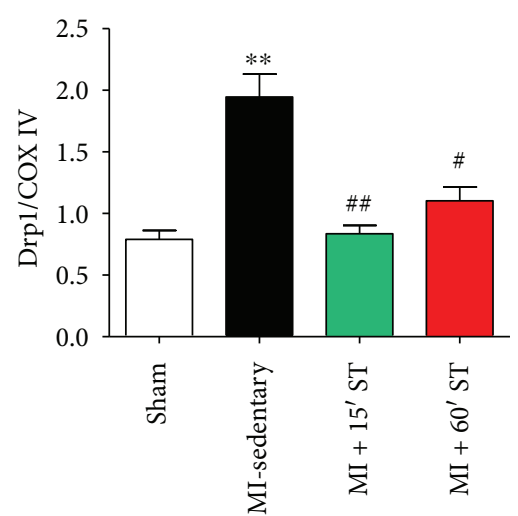

(e) (b)

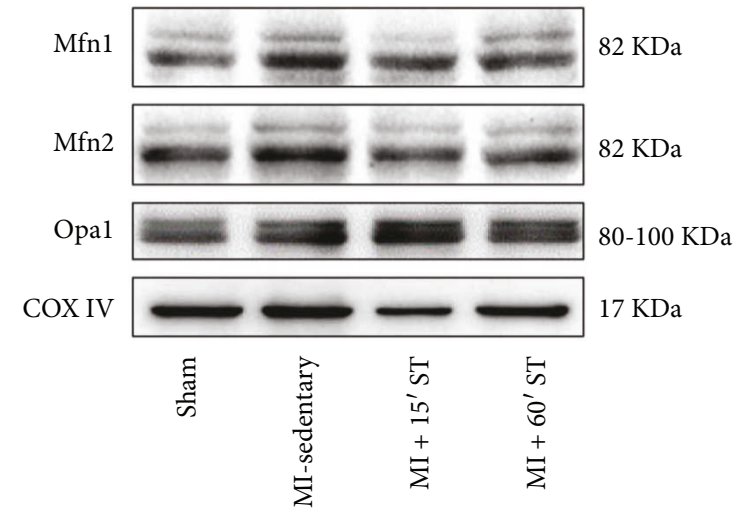

(d)

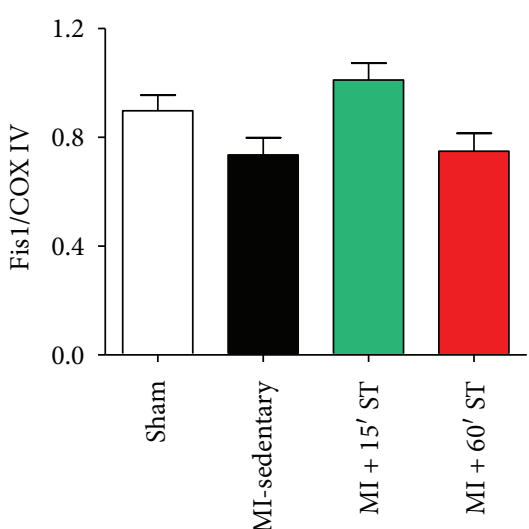

(f)

Figure 3: Continued. 


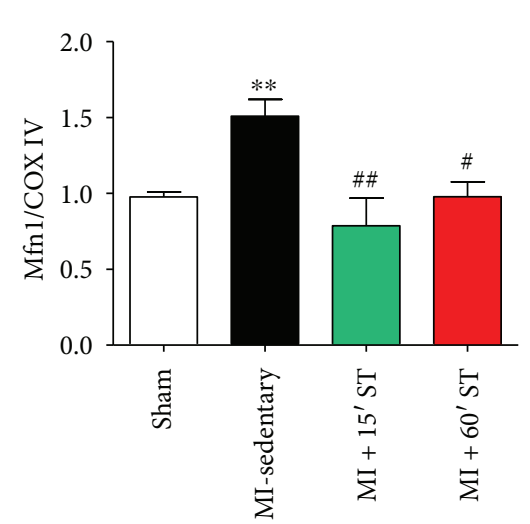

(g)

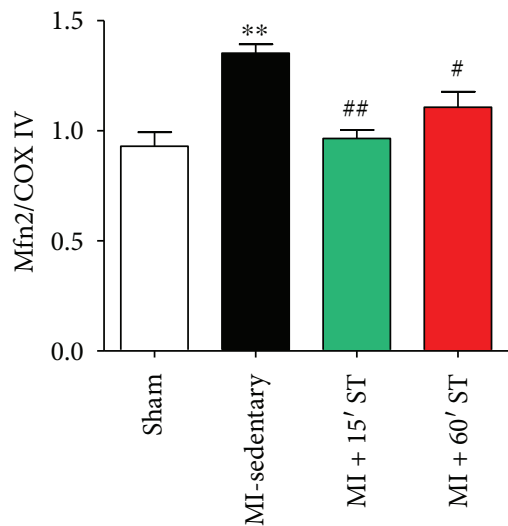

(h)

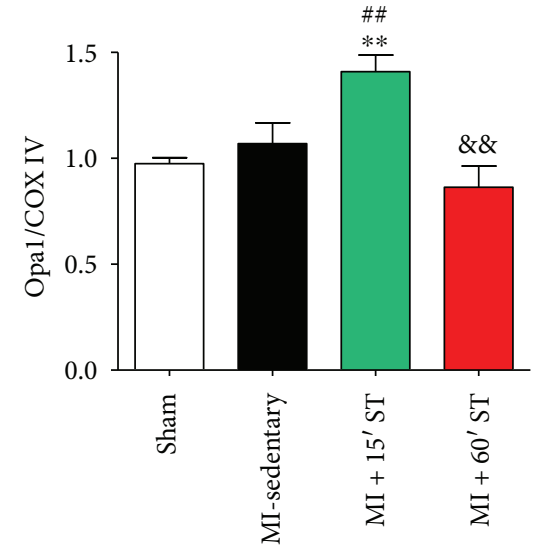

(i)

Figure 3: Short-duration exercise after MI modulates mitochondrial morphology and dynamics in aged heart. (a) Representative transmission electron micrographs of cardiac mitochondria. Scale bars, $1 \mu \mathrm{m}$. (b) Frequency distribution (\% total mitochondria) of the mitochondrial surface area. $N=243-251$. (c-i) Western blot analysis of mitochondrial fission and fusion markers. ${ }^{* *} P<0.01$ versus the sham group; ${ }^{\#} P<0.05,{ }^{\# \#} P<0.01$ versus the MI-sedentary group; ${ }^{\& \&} P<0.01$ versus the $\mathrm{MI}+15$ ' ST group. $N=6-8$.

TABLE 2: Mitochondrial morphological parameters of aged hearts after 8 weeks of swimming training.

\begin{tabular}{|c|c|c|c|c|}
\hline \multirow[b]{2}{*}{ Parameter } & \multicolumn{4}{|c|}{ Mean \pm SEM } \\
\hline & $\begin{array}{l}\text { Sham } \\
(n=243)\end{array}$ & $\begin{array}{l}\text { MI-sedentary } \\
\quad(n=257)\end{array}$ & $\begin{array}{c}\mathrm{MI}+15^{\prime} \mathrm{ST} \\
(n=240)\end{array}$ & $\begin{array}{c}\mathrm{MI}+60^{\prime} \mathrm{ST} \\
(n=251)\end{array}$ \\
\hline Area $\left(\mu \mathrm{m}^{2}\right)$ & $0.65 \pm 0.05$ & $1.17 \pm 0.12^{*}$ & $0.64 \pm 0.06^{\#}$ & $1.11 \pm 0.15^{*, \&}$ \\
\hline Aspect ratio & $1.97 \pm 0.11$ & $1.15 \pm 0.21^{* *}$ & $2.35 \pm 0.26^{*, \# \#}$ & $1.53 \pm 0.08^{*, \&}$ \\
\hline Circularity & $0.73 \pm 0.03$ & $0.88 \pm 0.03^{*}$ & $0.67 \pm 0.04$ & $0.77 \pm 0.02$ \\
\hline Roundness & $0.52 \pm 0.04$ & $0.73 \pm 0.05^{* *}$ & $0.50 \pm 0.05^{\#}$ & $0.68 \pm 0.04^{\&}$ \\
\hline Solidity & $0.97 \pm 0.01$ & $0.84 \pm 0.02$ & $0.91 \pm 0.01$ & $0.96 \pm 0.01$ \\
\hline
\end{tabular}

${ }^{*} P<0.05,{ }^{* *} P<0.01$ versus the sham group; ${ }^{\#} P<0.05,{ }^{\# \#} P<0.01$ versus the MI-sedentary group; ${ }^{8} P<0.05$ versus the MI +15 ' ST group. $N=243-257$.

25.8\% under hypoxia while in the SIRT3-ShRNA group, it significantly raised to $49.2 \%(P<0.05$, Figure $6(\mathrm{a}))$. Caspase- 3 activity manifested similar changes under SIRT3 deficiency $(P<0.01$, Figure 6(b)). Moreover, mitochondrial ROS production was increased in senescenced cardiomyocytes exposed to hypoxia. The inhibition of SIRT3 exacerbated superoxide generation compared to the $\mathrm{SI}+\mathrm{NC}$ group $(P<0.01$, Figures $6(\mathrm{c})-6(\mathrm{e}))$. The SOD2 activity was impaired by hypoxia and was even low with SIRT3 inhibition $(P<0.05$, Figure 6(f)). These results demonstrate that SIRT3 protects against SI injury in senescenced cardiomyocytes via antiapoptotic and antioxidative actions.

3.7. SIRT3 Regulates Mitochondrial Dynamics and Mitophagy Signaling under SI Injury. Finally, we measured mitochondrial dynamics and mitophagy signaling markers upon SIRT3 silencing. Compared to the SI + NC group, the level of Drp1 was significantly raised to $41.6 \% \quad(P<0.05$, Figures $7(\mathrm{a})$ and $7(\mathrm{~b}))$ and Opal expression was reduced $(P<0.05$, Figures $7(\mathrm{~d})$ and $7(\mathrm{~g}))$ under SIRT3 inhibition following hypoxia. However, no obvious changes were observed on Mfn1, Mfn2, and Fis1 (Figures 7(a) and 7(c)7(f)) expression upon SIRT3 silencing. SIRT3 deficiency manifested the upregulation of LC3-II $(P<0.05)$ and P62 levels $(P<0.05)$ compared to the $\mathrm{SI}+\mathrm{NC}$ group (Figures 8(a)-8(c)). Meanwhile, SIRT3 deficiency suppressed PINK1 and Parkin expression under hypoxia $(P<0.05$, $P<0.01$, resp., Figures $8(\mathrm{~d})$ and $8(\mathrm{e}))$, demonstrating that the inhibition of SIRT3 blunted mitophagy progress under hypoxia.

\section{Discussion}

Cardiac remodeling following MI is associated with left ventricular dysfunction, cardiac hypertrophy, myocardial interstitial fibrosis, and cardiomyocyte apoptosis, which finally lead to decompensated heart failure [28]. Exercisebased cardiac rehabilitation is an effective therapy in attenuating post-MI remodeling [2, 29]. However, some studies exhibited that training with a high volume (duration) led to a lower all-cause mortality $[5,30]$ while others displayed excessive exercise significantly increased cardiovascular mortality [6]. The proper exercise duration, especially for aged patients with $\mathrm{CHD}$, remains as a question of debate $[31,32]$. Furthermore, the underlying molecular mechanism is still unclear. 


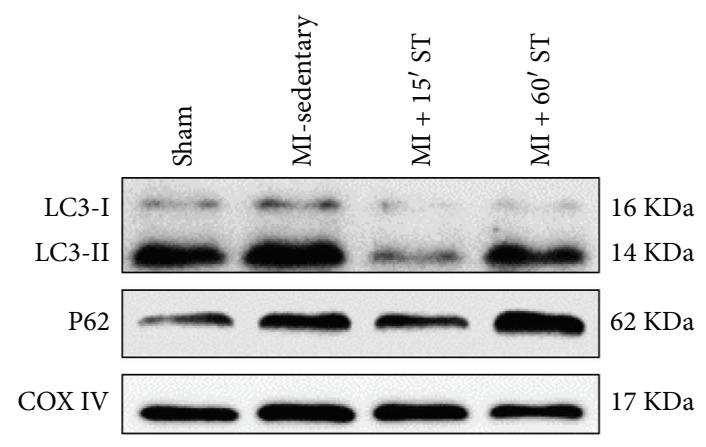

(a)

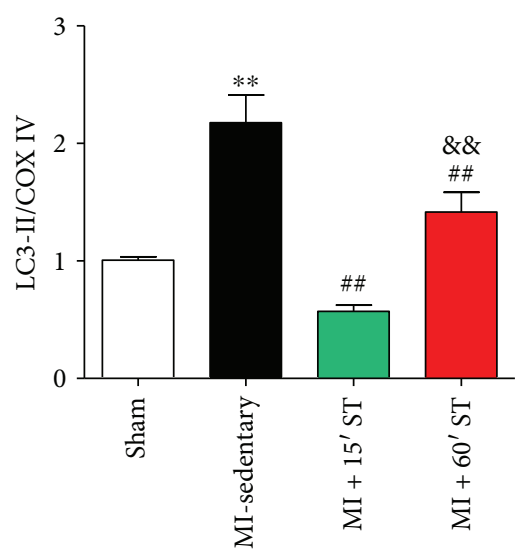

(b)
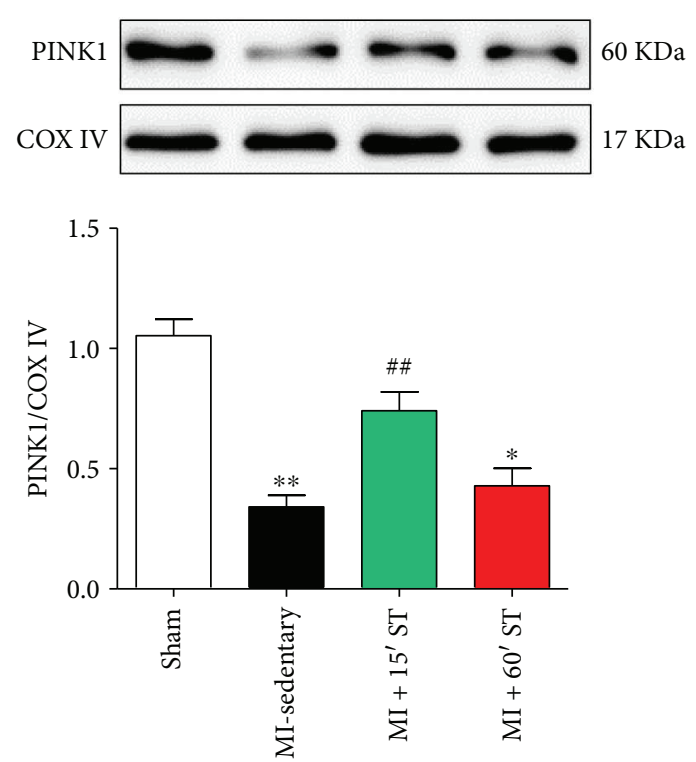

(d)

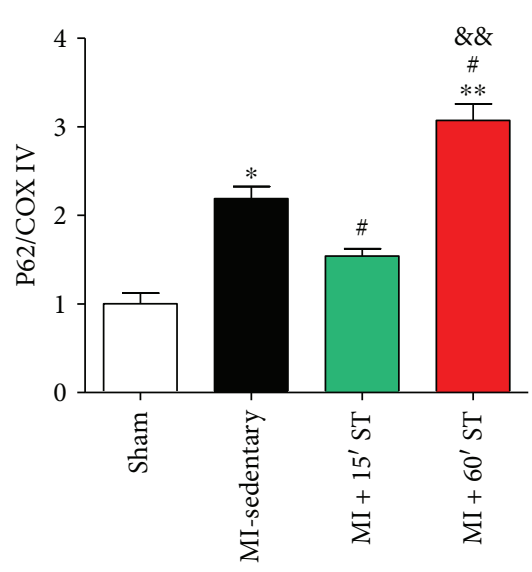

(c)
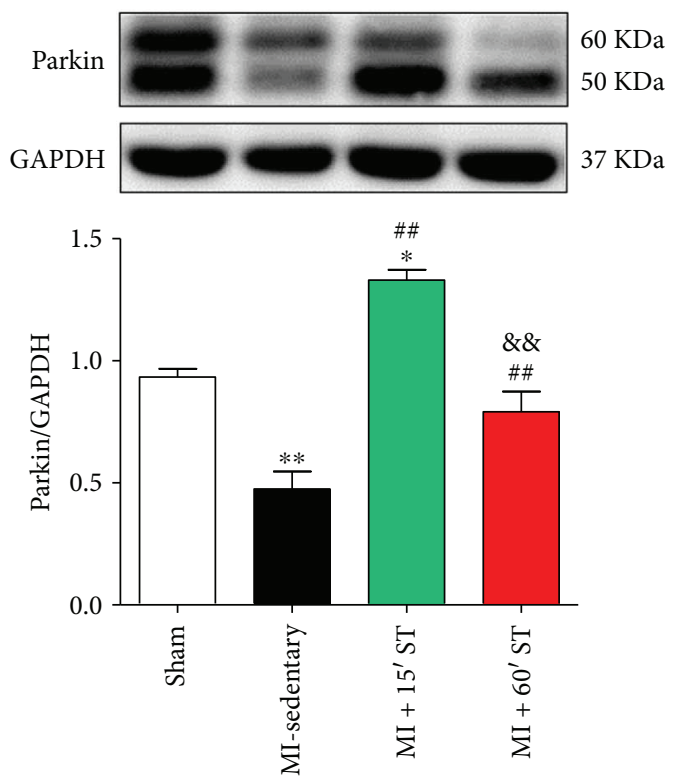

(e)

FIGURE 4: Short-duration exercise after MI regulates mitophagy signaling in aged mice. (a-c) Western blot analysis of mitophagy markers LC3 and P62. (d-e) Western blot analysis of PINK1 and Parkin. ${ }^{*} P<0.05,{ }^{* *} P<0.01$ versus the sham group; ${ }^{\#} P<0.05$, \#\# $P<0.01$ versus the MI-sedentary group; ${ }^{\& \&} P<0.01$ versus the $\mathrm{MI}+15$ ST group. $N=6-8$.

ST presents a better redistribution of blood flow without significant variations in the cardiac output and heart rate than treadmill or voluntary wheel running programs [33]. Moreover, swimming is much easier for the elderly joints due to reduced effects of gravity. In this study, we assessed two different durations ( $75 \mathrm{~min} /$ week and $300 \mathrm{~min} /$ week) of ST's rehabilitative effects on aged mice following stable MI and demonstrated for the first time that short-duration rather than long-duration exercise after $\mathrm{MI}$ attenuated cardiac dysfunction and improved the survival rate in aged 

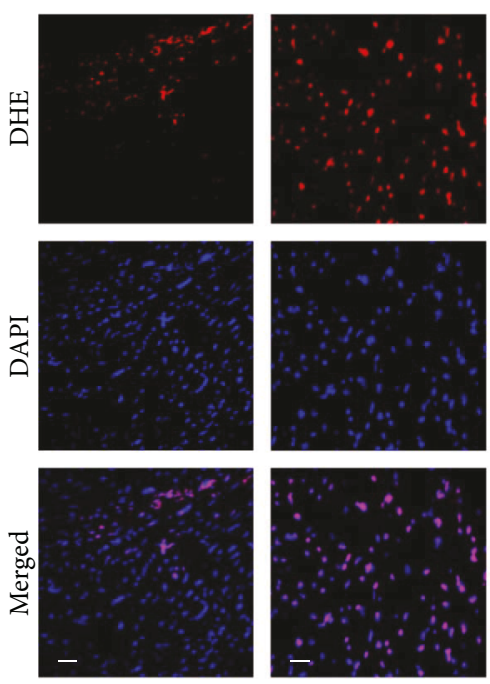

Sham

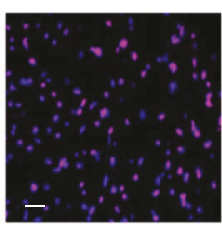

MI-sedentary
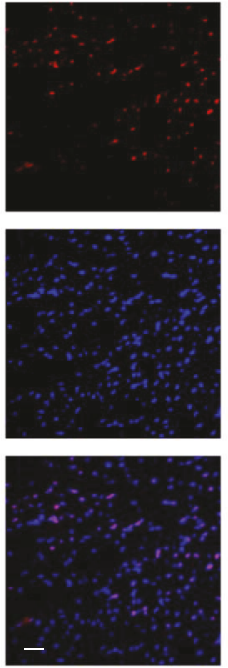

$\mathrm{MI}+15^{\prime} \mathrm{ST}$
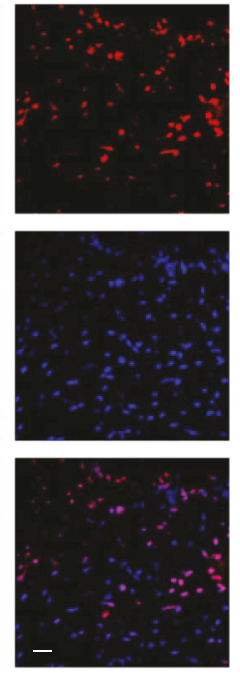

$\mathrm{MI}+60^{\prime} \mathrm{ST}$

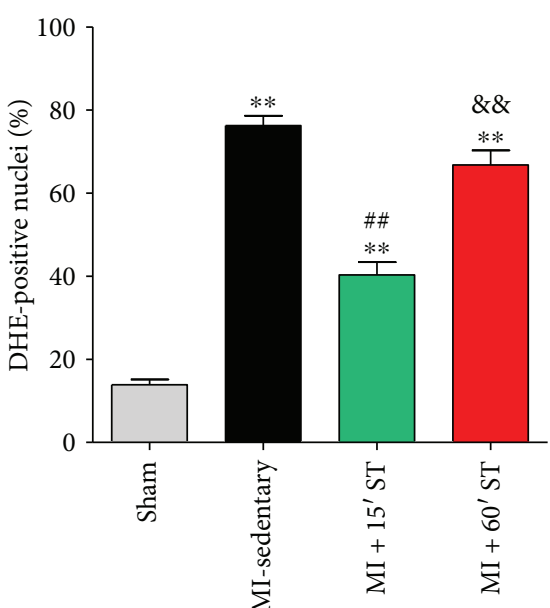

(b)
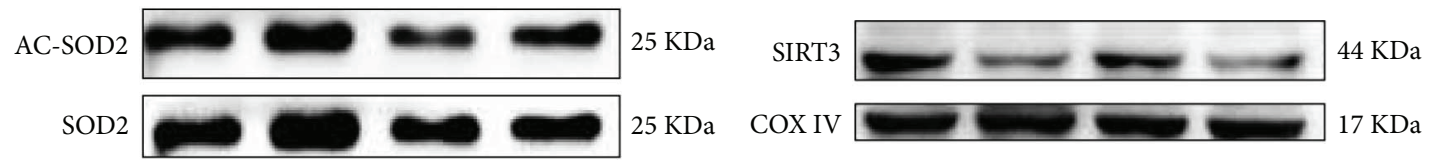

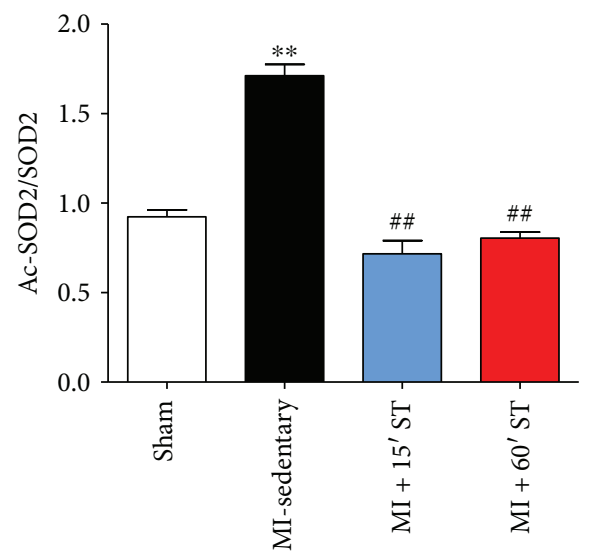

(c)

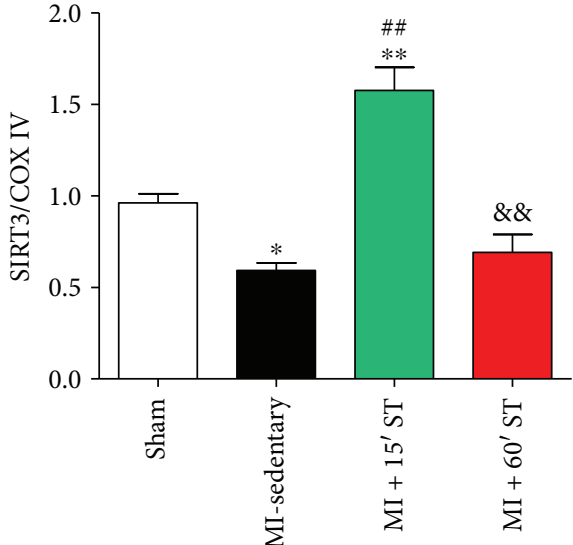

(d)

FIGURE 5: Short-duration exercise after MI attenuates oxidative stress and increases SIRT3 expression in aged heart. (a) ROS production determined by DHE staining. Scale bars, $50 \mu \mathrm{m}$. (b) Statistical analysis of DHE positive cells. (c) Ac-SOD2 to SOD2 ratio. (d) Western blot analysis of mitochondrial SIRT3. ${ }^{*} P<0.05$; ${ }^{* *} P<0.01$ versus the sham group; ${ }^{\# \# ~} P<0.01$ versus the MI-sedentary group; \&\& $P<0.01$ versus the $\mathrm{MI}+15^{\prime} \mathrm{ST}$ group. $N=6-8$.

mice via inhibition of myocardial fibrosis and apoptosis. We suggest that the discrepancies result on exercise duration from several factors. First, clinical studies using a questionnaire have a certain degree of limitation, including undetailed exercise interventions and the subjectivity of the reports. Patients' baseline differences and societal factors also vary from different studies [5]. Moreover, advanced age contributes to a general trend towards the decrease in exercise tolerance [34].

Mitochondria are deeply involved in the aging process [35]. Previous studies suggest a causative link between mitochondrial dysfunction and major phenotypes associated with aging $[36,37]$. Aged mitochondria are more susceptible to ischemia injury while regular exercise is demonstrated to improve mitochondrial quality and quantity $[10,38]$. In the current study, we observed impaired mitochondrial morphology in aged mice post-MI. A significant increase of swollen mitochondria contributes to the larger mitochondrial surface area while MI-induced excessive mitochondrial fission may lead to the increase of small, round mitochondria. Our data revealed that short-duration exercise improved mitochondrial network remodeling via regulation 


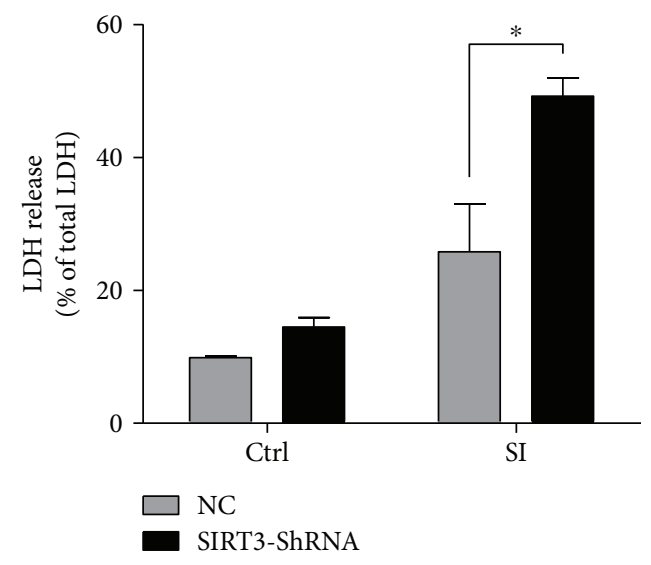

(a)

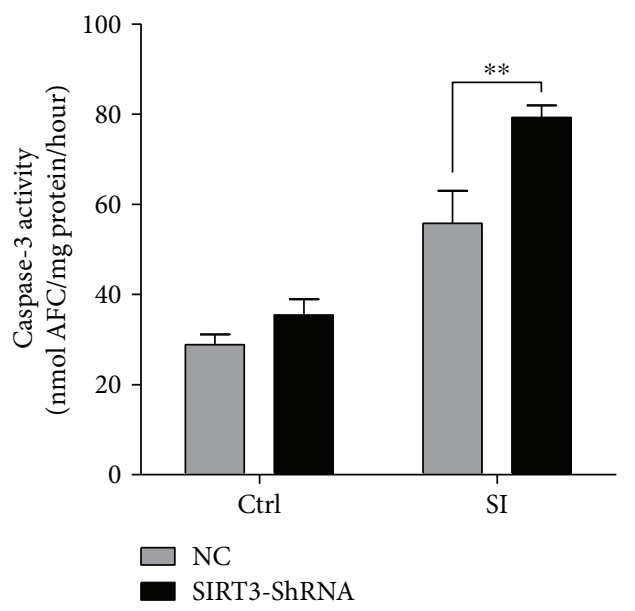

(b)
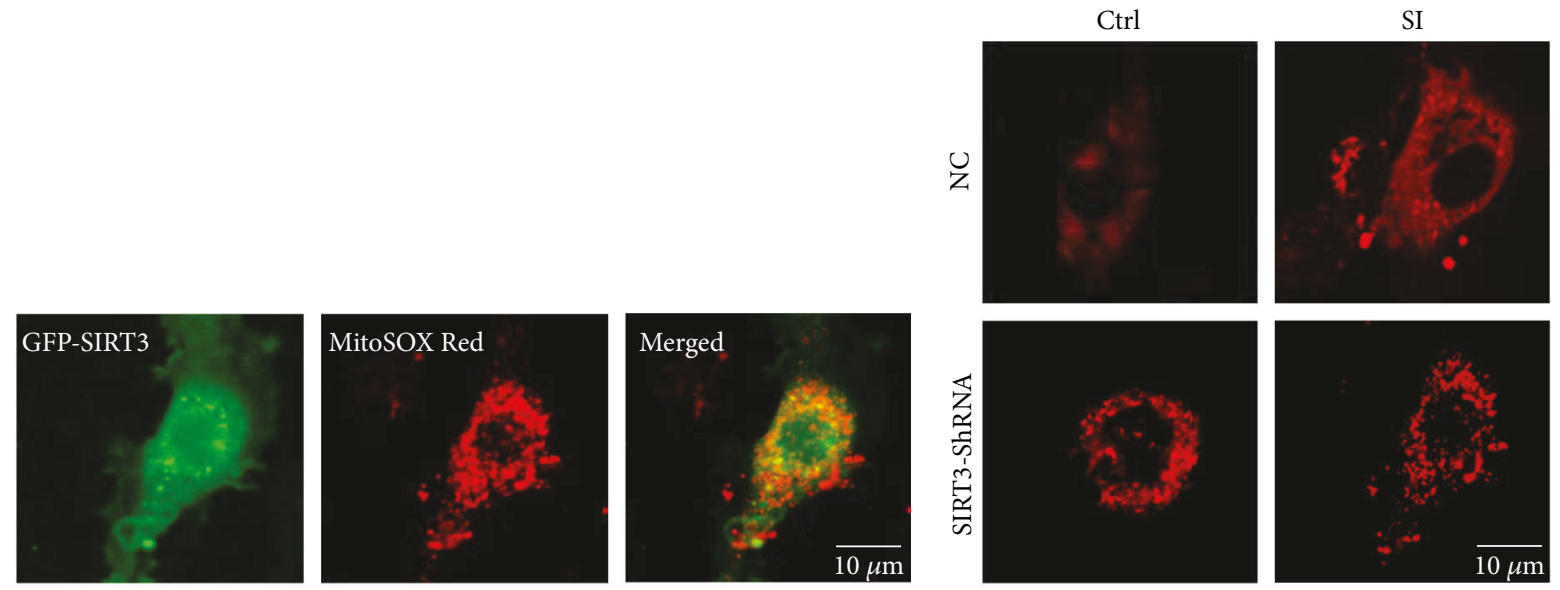

(c)
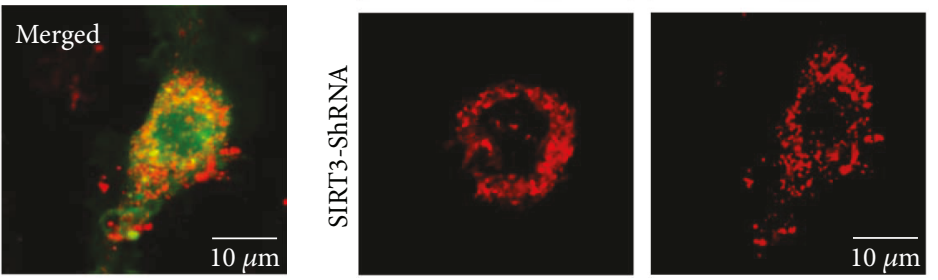

(d)
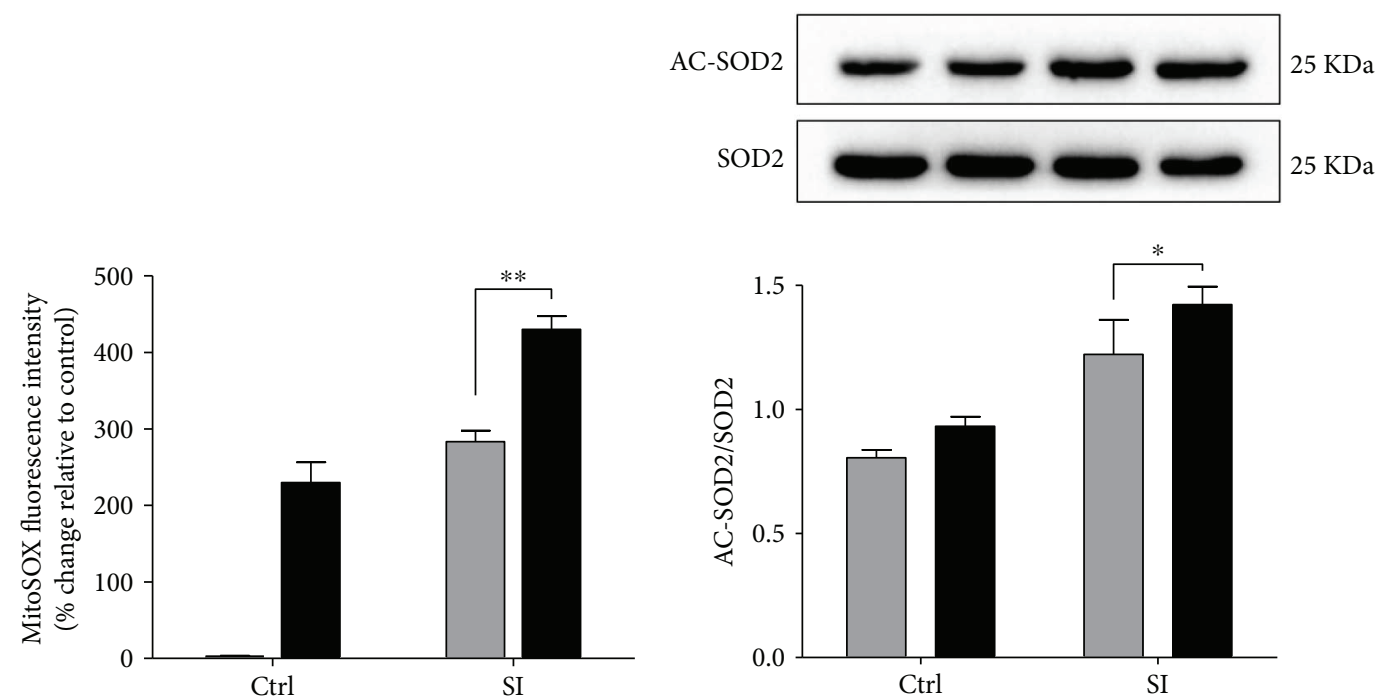

NC

SIRT3-ShRNA

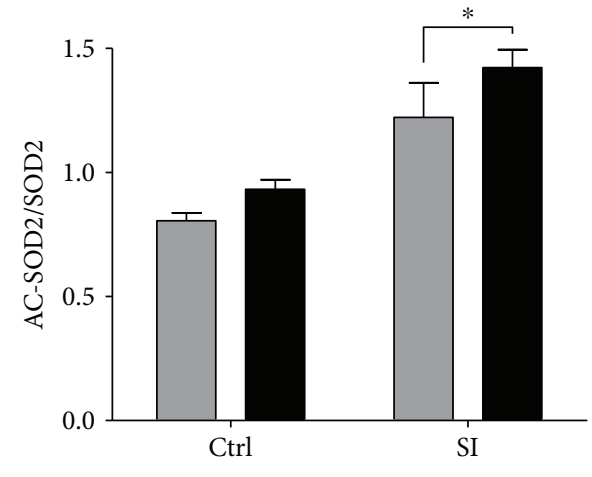

$\square \mathrm{NC}$

- SIRT3-ShRNA

(f)

FIGURE 6: SIRT3 deficiency exacerbates SI-induced cardiomyocyte apoptosis and oxidative stress. (a) Effect of SIRT3 silencing on cell death, determined by LDH release assay. (b) Effect of SIRT3 silencing on apoptotic events, determined by caspase-3 activity assay. (c-e) Effect of SIRT3 silencing on mitochondrial ROS production, determined by MitoSOX Red dye. Scale bars, $10 \mu \mathrm{m}$. (f) Ac-SOD2 to SOD2 ratio upon SIRT3 inhibition. ${ }^{*} P<0.05,{ }^{* *} P<0.01$ versus the SI + NC group. $N=3-6$. 


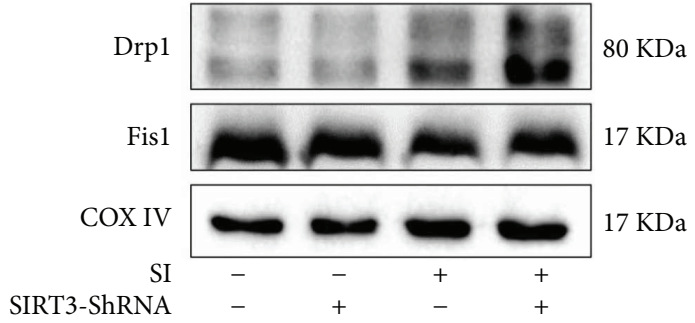

(a)

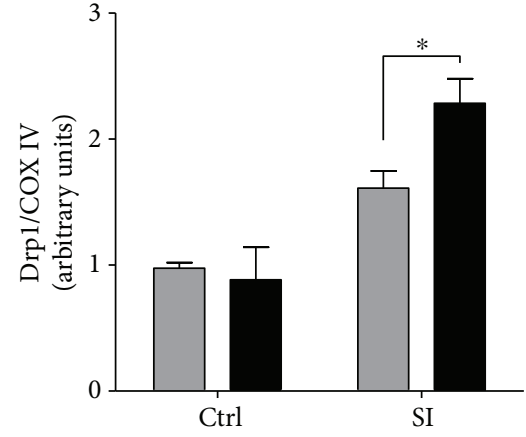

$\square \mathrm{NC}$

- SIRT3-ShRNA

(b)

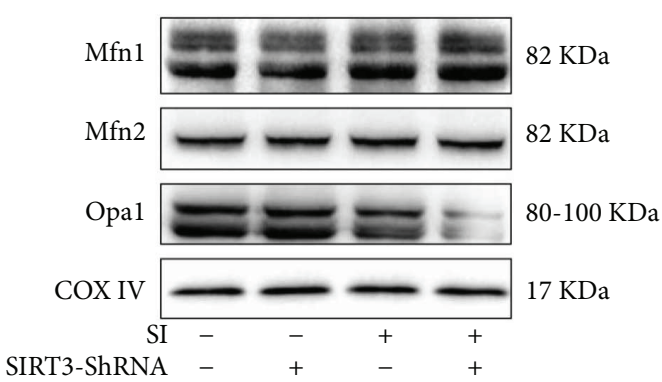

(d)

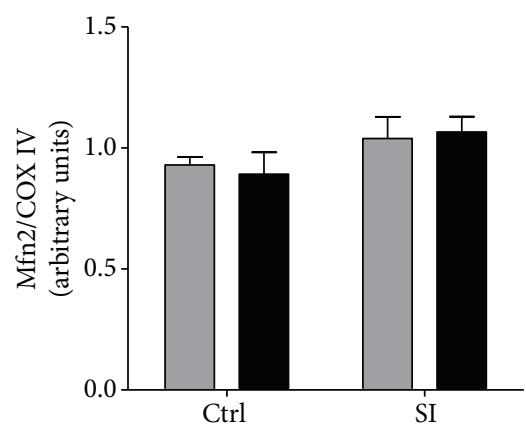

$\square$ NC

- SIRT3-ShRNA

(f)

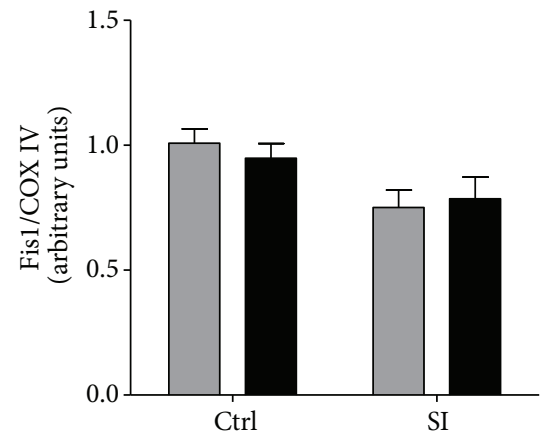

$\square \mathrm{NC}$

- SIRT3-ShRNA

(c)

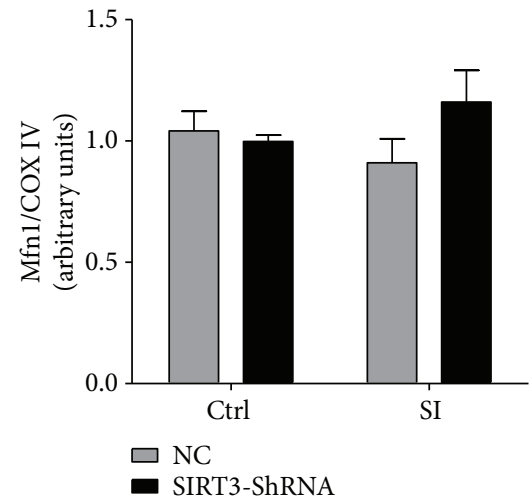

(e)

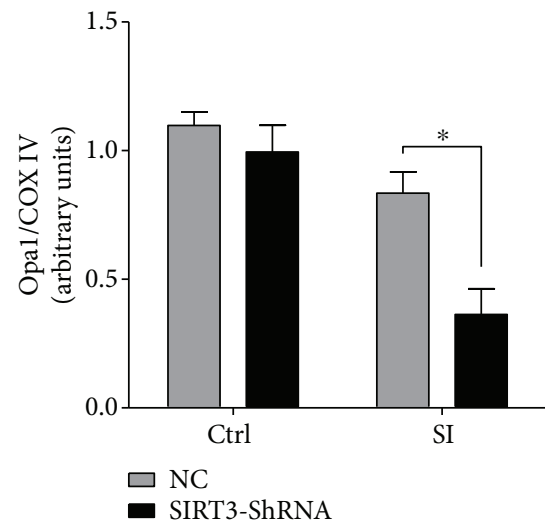

(g)

FIGURE 7: SIRT3 regulates cardiomyocytes mitochondrial dynamics under SI injury. (a-c) Western blot analysis of fission proteins Drp1 and Fis1 upon SIRT3 silencing. (d-g) Western blot analysis of fusion proteins Mfn1, Mfn2, and Opa1 upon SIRT3 silencing. ${ }^{*} P<0.05$ versus the $\mathrm{SI}+\mathrm{NC}$ group. $\mathrm{N}=3-6$. 


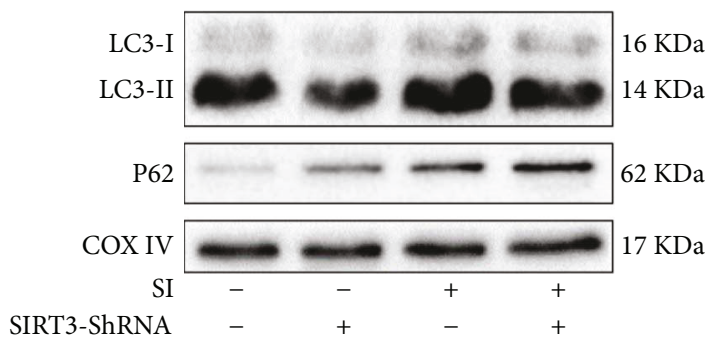

(a)

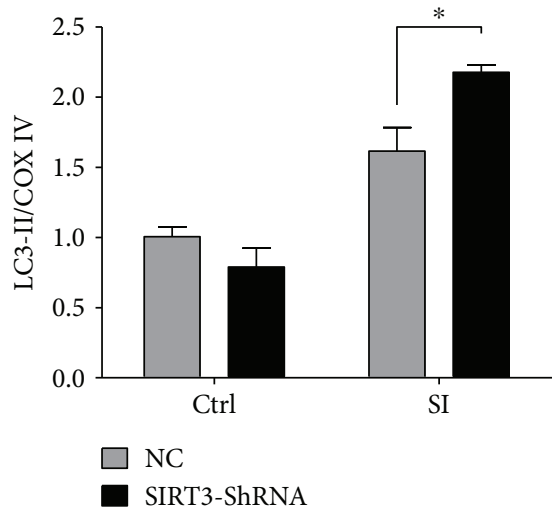

(b)

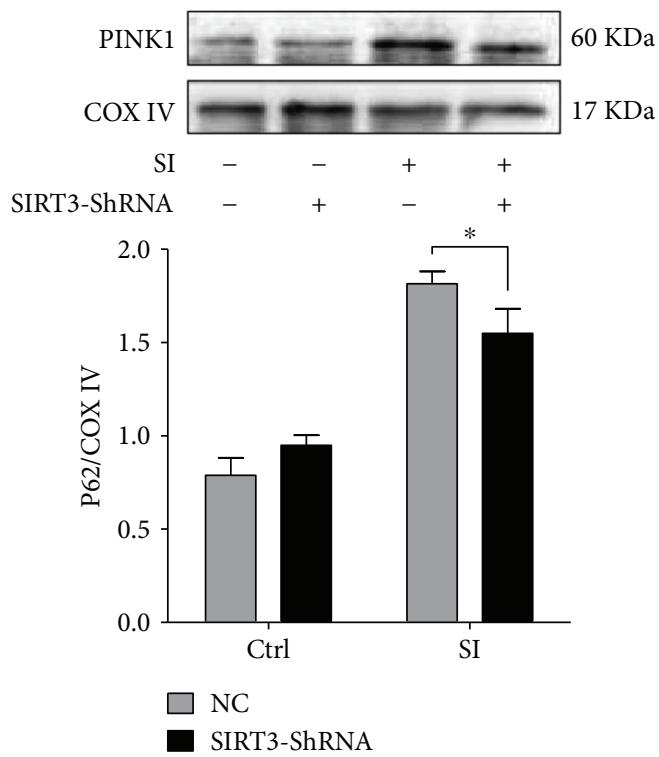

(d)

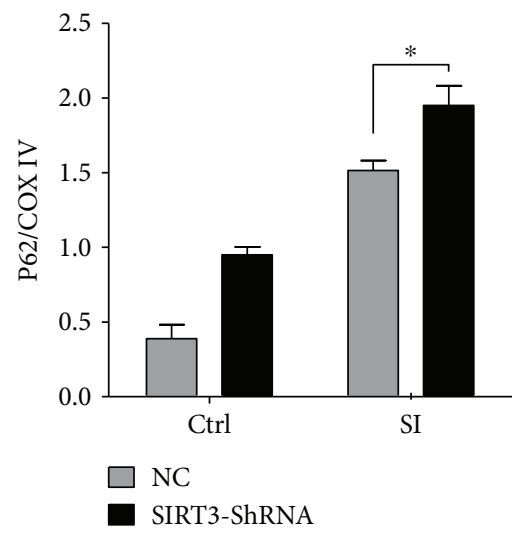

(c)

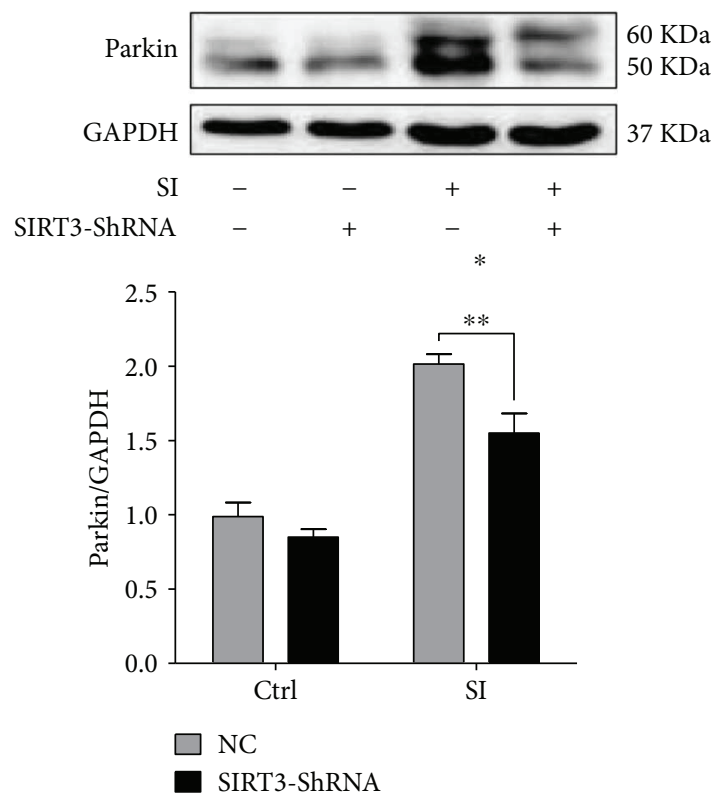

(e)

FIGURE 8: SIRT3 regulates SI-induced mitophagy signaling in vitro. $(\mathrm{a}-\mathrm{c})$ Western blot analysis of mitophagy makers LC3 and P62 upon SIRT3 silencing. (d-e) Western blot analysis of PINK1 and Parkin upon SIRT3 silencing. ${ }^{*} P<0.05,{ }^{* *} P<0.01$ versus the SI + NC group. $N=3-6$.

of mitochondrial dynamics signaling-related proteins Drp1 and Opa1. Mitochondrial fragmentation results in loss of ATP synthesis, increased ROS production, and release of proapoptotic proteins, including cytochrome $c$ and BAX [39]. Exercise-stimulated inhibition of Drp1 restores mitochondrial morphology and promotes cardiac function after MI [40, 41] probably due to increased mPTP resistance [42]. Meanwhile, enhancement of Opa1 maintains morphology of cristae and regulates mitochondrial metabolism since Opa1 protects against apoptotic signaling via preventing cytochrome $\mathrm{c}$ release independently from mitochondrial fusion $[43,44]$. Studies demonstrated that the mutation of Opa1 is associated with reduced oxidative phosphorylation and ATP synthesis in skeletal muscle and human fibroblasts $[45,46]$.

Next, we found that aged hearts after MI perform an upregulation of mitophagy signaling-related protein LC3-II 
and P62 levels together with the inhibition of PINK1/Parkin expression. PINK1 is stabilized and recruits Parkin to mitochondria, initiating mitophagy [47]. The low expression of PINK1/Parkin suggests that the accumulation of LC3-II and P62 protein levels is from inhibition of LC3II turnover, implicating a block either in autophasosome/ lysosome assembling or autolysosome degradation. Our data revealed that short-duration exercise regulates mitophagy signaling biomarkers, including activating PINK1/Parkin expression together with decreasing LC3-II and P62 levels.

Moreover, we demonstrated that aged hearts after MI perform increased oxidative stress with accumulated ROS production and reduced antioxidant enzyme SOD2 activity, consistent with another study [48]. MI-induced ROS accumulation may further contribute to our findings of the increased mitochondrial fragmentation and impaired mitochondrial quality control. Our data revealed that shortduration exercise reduces ROS production and enhances the activity of antioxidant enzyme SOD2 by inhibiting its acetylation in aged mice after MI. The inhibition of oxidative stress directly attenuates cardiac fibrosis and apoptosis to protect against post-MI injury.

Finally, we proved that short-duration exercise after MI upregulates mitochondrial SIRT3 expression in aged mice while SIRT3 deficiency exacerbates cardiomyocyte oxidative stress and apoptosis and blocks mitochondrial dynamics and mitophagy signaling under hypoxia in vitro. SIRT3 regulates mitochondrial biogenesis and function and promotes mitochondrial oxidative stress resistance via altering the acetylation level of MnSOD and enhancing its ability to scavenge ROS [49]. In particular, decreased SIRT3 contributes to the susceptibility of aged hearts to myocardial ischemia/reperfusion (MI/R) injury [50]. Previous studies showed that exercise training increases SIRT3 expression in skeletal muscle [18]. However, Karvinen et al. revealed that high capacity running rats had a higher SIRT3 protein content in skeletal muscle compared to low capacity running rats, contributing to lower mitochondrial protein acetylation [51]. The discrepancies between our finding and Karvinen et al.'s finding may attribute to different exercise models.

In conclusion, different durations of exercise may stimulate different mechanical effects. Exercise duration has independent effects on the hormonal response that significantly affects systemic metabolism [52]. Short-duration exercise elicits a great reduction of serum insulin concentrations and insulin resistance [53] while long-duration exercise increases adrenocorticotropic hormone (ACTH), cortisol, and growth hormone [54]. Studies found that long-duration exercise produces a prolonged postexercise oxygen consumption (EPOC), elevating the energy cost [55]. Our study demonstrated for the first time that sustained short-duration ST after MI attenuates cardiac dysfunction in aged mice. It is likely that the positive regulation induced by a short-duration ST regimen on the elevated SIRT3 protein level improved mitochondrial quality control and decreased apoptosis and fibrosis contributed to the observed more resistant phenotype. However, long-duration ST elicits a deleterious response on CR that may attribute to the declined exercise tolerance in aged mice.

\section{Limitation}

Considering exercising condition is hard to mimic in vitro, we only examined the role of SIRT3 on the regulation of mitochondrial quality control and apoptosis in senescent $\mathrm{H} 9 \mathrm{c} 2$ cells under hypoxia. SIRT3-knockout mice may be used in further studies to confirm the relationship between SIRT3 and exercise's cardioprotective effects.

\section{Conflicts of Interest}

The authors declare no conflicts of interest.

\section{Authors' Contributions}

Dajun Zhao, Yang Sun, Yanzhen Tan, and Zhengbin Zhang contributed equally to this work.

\section{Acknowledgments}

This work was supported by the National High-Tech R\&D Program of China (2015AA020919) and Natural Science Foundation of China (81422004, 81470480, and 81470477).

\section{Supplementary Materials}

Supplementary 1. H9c2 cardiomyocyte senescence and lentivirus-mediated SIRT3 silencing in vitro. (A) Representative images (magnification, $\times 100$ ) and quantitation of senescence-associated- $\beta$-galactosidase (SA- $\beta$-gal) activity in cardiomyocytes treated with doxorubicin (DOX, $0.1 \mu \mathrm{M}$ ). Scale bars, $50 \mu \mathrm{m}$. (B) Representative images showing the morphological changes and SA- $\beta$-gal activity in cardiomyocytes treated with $0.1 \mu \mathrm{M}$ DOX. Cells were counterstained with Giemsa solution (magnification, $\times 400$ ). Scale bars, $10 \mu \mathrm{m}$. (C) Representative images showing the infection of GFP-lentivirus-SIRT3-ShRNA. Scale bars, $100 \mu \mathrm{m}$. (D) Efficiency of SIRT3 silencing in vitro determined by representative Western blot. (E) Cell viability determined by CCK-8 assay. ${ }^{* *} P<0.01$ versus the NC group. $N=3-6$.

Supplementary 2. Exercise's effects on mitochondrial biogenesis and matrix markers. (A-C) Western blot analysis of PGC1- $\alpha$ and HSP60. ${ }^{* *} P<0.01$ versus the sham group; ${ }^{\#} P<0.01$ versus the MI-sedentary group. $N=6$.

\section{References}

[1] P. A. Ades, "Cardiac rehabilitation and secondary prevention of coronary heart disease," New England Journal of Medicine, vol. 345, no. 12, pp. 892-902, 2001.

[2] R. S. Taylor, A. Brown, S. Ebrahim et al., "Exercise-based rehabilitation for patients with coronary heart disease: systematic review and meta-analysis of randomized controlled trials," The American Journal of Medicine, vol. 116, no. 10, pp. 682692, 2004.

[3] P. D. Thompson, D. Buchner, I. L. Pina et al., "Exercise and physical activity in the prevention and treatment of atherosclerotic cardiovascular disease: a statement from the council on clinical cardiology (subcommittee on exercise, rehabilitation, and prevention) and the council on nutrition, physical activity, 
and metabolism (subcommittee on physical activity)," Circulation, vol. 107, no. 24, pp. 3109-3116, 2003.

[4] D. Acanfora, P. Scicchitano, G. Casucci et al., "Exercise training effects on elderly and middle-age patients with chronic heart failure after acute decompensation: a randomized, controlled trial," International Journal of Cardiology, vol. 225, pp. 313-323, 2016.

[5] R. A. H. Stewart, C. Held, N. Hadziosmanovic et al., "Physical activity and mortality in patients with stable coronary heart disease," Journal of the American College of Cardiology, vol. 70, no. 14, pp. 1689-1700, 2017.

[6] P. T. Williams and P. D. Thompson, "Increased cardiovascular disease mortality associated with excessive exercise in heart attack survivors," Mayo Clinic Proceedings, vol. 89, no. 9, pp. 1187-1194, 2014.

[7] D. R. Green, L. Galluzzi, and G. Kroemer, "Mitochondria and the autophagy-inflammation-cell death axis in organismal aging," Science, vol. 333, no. 6046, pp. 1109-1112, 2011.

[8] H. E. Collins, L. He, L. Zou et al., "Stromal interaction molecule 1 is essential for normal cardiac homeostasis through modulation of ER and mitochondrial function," American Journal of Physiology-Heart and Circulatory Physiology, vol. 306, no. 8, pp. H1231-H1239, 2014.

[9] Y. Ikeda, A. Shirakabe, Y. Maejima et al., "Endogenous Drp1 mediates mitochondrial autophagy and protects the heart against energy stress," Circulation Research, vol. 116, no. 2, pp. 264-278, 2015.

[10] Z. Yan, V. A. Lira, and N. P. Greene, "Exercise traininginduced regulation of mitochondrial quality," Exercise and Sport Sciences Reviews, vol. 40, no. 3, pp. 159-164, 2012.

[11] S. Larsen, S. Skaaby, J. W. Helge, and F. Dela, "Effects of exercise training on mitochondrial function in patients with type 2 diabetes," World Journal of Diabetes, vol. 5, no. 4, pp. 482-492, 2014.

[12] L. Luo, J.-R. Dai, S.-S. Guo et al., "Lysosomal proteolysis is associated with exercise-induced improvement of mitochondrial quality control in aged hippocampus," The Journals of Gerontology: Series A, vol. 72, no. 10, pp. 1342-1351, 2017.

[13] S. Winnik, J. Auwerx, D. A. Sinclair, and C. M. Matter, "Protective effects of sirtuins in cardiovascular diseases: from bench to bedside," European Heart Journal, vol. 36, no. 48, pp. 3404-3412, 2015.

[14] A. S. Bause and M. C. Haigis, "SIRT3 regulation of mitochondrial oxidative stress," Experimental Gerontology, vol. 48, no. 7, pp. 634-639, 2013.

[15] A. Giralt and F. Villarroya, "SIRT3, a pivotal actor in mitochondrial functions: metabolism, cell death and aging," Biochemical Journal, vol. 444, no. 1, pp. 1-10, 2012.

[16] T. Bochaton, C. Crola-Da-Silva, B. Pillot et al., "Inhibition of myocardial reperfusion injury by ischemic postconditioning requires sirtuin 3-mediated deacetylation of cyclophilin D," Journal of Molecular and Cellular Cardiology, vol. 84, pp. 6169, 2015.

[17] X. Hou, H. Zeng, X. He, and J. X. Chen, "Sirt3 is essential for apelin-induced angiogenesis in post-myocardial infarction of diabetes," Journal of Cellular and Molecular Medicine, vol. 19, no. 1, pp. 53-61, 2015.

[18] O. M. Palacios, J. J. Carmona, S. Michan et al., "Diet and exercise signals regulate SIRT3 and activate AMPK and PGC-1 $\alpha$ in skeletal muscle," Aging, vol. 1, no. 9, pp. 771-783, 2009.
[19] E. Gao, Y. H. Lei, X. Shang et al., "A novel and efficient model of coronary artery ligation and myocardial infarction in the mouse," Circulation Research, vol. 107, no. 12, pp. 1445$1453,2010$.

[20] Q. X. Li, Z. Y. Xiong, B. P. Hu et al., "Aging-associated insulin resistance predisposes to hypertension and its reversal by exercise: the role of vascular vasorelaxation to insulin," Basic Research in Cardiology, vol. 104, no. 3, pp. 269-284, 2009.

[21] Q.-J. Zhang, Q.-X. Li, H.-F. Zhang et al., "Swim training sensitizes myocardial response to insulin: role of Akt-dependent eNOS activation," Cardiovascular Research, vol. 75, no. 2, pp. 369-380, 2007.

[22] P. Spallarossa, P. Altieri, C. Aloi et al., "Doxorubicin induces senescence or apoptosis in rat neonatal cardiomyocytes by regulating the expression levels of the telomere binding factors 1 and 2," American Journal of PhysiologyHeart and Circulatory Physiology, vol. 297, no. 6, pp. H2169H2181, 2009.

[23] Y. Maejima, S. Adachi, H. Ito, K. Hirao, and M. Isobe, "Induction of premature senescence in cardiomyocytes by doxorubicin as a novel mechanism of myocardial damage," Aging Cell, vol. 7, no. 2, pp. 125-136, 2008.

[24] G. P. Dimri, X. Lee, G. Basile et al., "A biomarker that identifies senescent human cells in culture and in aging skin in vivo," Proceedings of the National Academy of Sciences of the United States of America, vol. 92, no. 20, pp. 9363-9367, 1995.

[25] L. Liu, Y. Wang, Z. Y. Cao et al., "Up-regulated TLR4 in cardiomyocytes exacerbates heart failure after long-term myocardial infarction," Journal of Cellular and Molecular Medicine, vol. 19, no. 12, pp. 2728-2740, 2015.

[26] X. Sun, A. Momen, J. Wu et al., "p27 protein protects metabolically stressed cardiomyocytes from apoptosis by promoting autophagy," Journal of Biological Chemistry, vol. 289, no. 24, pp. 16924-16935, 2014.

[27] Y. Wang, X. Wang, J. F. Jasmin et al., "Essential role of caveolin-3 in adiponectin signalsome formation and adiponectin cardioprotection," Arteriosclerosis, Thrombosis, and Vascular Biology, vol. 32, no. 4, pp. 934-942, 2012.

[28] J. N. Cohn, R. Ferrari, and N. Sharpe, "Cardiac remodelingconcepts and clinical implications: a consensus paper from an international forum on cardiac remodeling," Journal of the American College of Cardiology, vol. 35, no. 3, pp. 569$582,2000$.

[29] L. Anderson, N. Oldridge, D. R. Thompson et al., "Exercisebased cardiac rehabilitation for coronary heart disease: Cochrane systematic review and meta-analysis," Journal of the American College of Cardiology, vol. 67, no. 1, pp. 112, 2016.

[30] L. Anderson and R. S. Taylor, "Cardiac rehabilitation for people with heart disease: an overview of Cochrane systematic reviews," Cochrane Database of Systematic Reviews, 2014.

[31] M. Volterrani and F. Iellamo, "Cardiac rehabilitation in patients with heart failure - new perspectives in exercise training," Cardiac Failure Review, vol. 2, no. 1, pp. 63-68, 2016.

[32] P. Parto, J. H. O'Keefe, and C. J. Lavie, "The exercise rehabilitation paradox: less may be more?," The Ochsner Journal, vol. 16, no. 3, pp. 297-303, 2016.

[33] Y. Wang, U. Wisloff, and O. J. Kemi, "Animal models in the study of exercise-induced cardiac hypertrophy," Physiological Research, vol. 59, no. 5, pp. 633-644, 2010. 
[34] D. W. Schopfer and D. E. Forman, "Cardiac rehabilitation in older adults," Canadian Journal of Cardiology, vol. 32, no. 9, pp. 1088-1096, 2016.

[35] C. Lopez-Otin, M. A. Blasco, L. Partridge, M. Serrano, and G. Kroemer, "The hallmarks of aging," Cell, vol. 153, no. 6, pp. 1194-1217, 2013.

[36] J. F. Passos, G. Saretzki, and T. von Zglinicki, "DNA damage in telomeres and mitochondria during cellular senescence: is there a connection?," Nucleic Acids Research, vol. 35, no. 22, pp. 7505-7513, 2007.

[37] A. Bratic and N. G. Larsson, "The role of mitochondria in aging," Journal of Clinical Investigation, vol. 123, no. 3, pp. 951-957, 2013.

[38] S. K. Powers, K. J. Sollanek, M. P. Wiggs, H. A. Demirel, and A. J. Smuder, "Exercise-induced improvements in myocardial antioxidant capacity: the antioxidant players and cardioprotection," Free Radical Research, vol. 48, no. 1, pp. 43-51, 2014.

[39] S. Bialik, V. L. Cryns, A. Drincic et al., "The mitochondrial apoptotic pathway is activated by serum and glucose deprivation in cardiac myocytes," Circulation Research, vol. 85, no. 5, pp. 403-414, 1999.

[40] M.-H. Disatnik, J. C. B. Ferreira, J. C. Campos et al., “Acute inhibition of excessive mitochondrial fission after myocardial infarction prevents long-term cardiac dysfunction," Journal of the American Heart Association, vol. 2, no. 5, article e000461, 2013.

[41] H. K. Jiang, Y. H. Wang, L. Sun et al., "Aerobic interval training attenuates mitochondrial dysfunction in rats postmyocardial infarction: roles of mitochondrial network dynamics," International Journal of Molecular Sciences, vol. 15, no. 12, pp. 5304-5322, 2014.

[42] T. Yu, S. S. Sheu, J. L. Robotham, and Y. Yoon, "Mitochondrial fission mediates high glucose-induced cell death through elevated production of reactive oxygen species," Cardiovascular Research, vol. 79, no. 2, pp. 341-351, 2008.

[43] C. Frezza, S. Cipolat, O. Martins de Brito et al., "OPA1 controls apoptotic cristae remodeling independently from mitochondrial fusion," Cell, vol. 126, no. 1, pp. 177-189, 2006.

[44] A. Olichon, L. Baricault, N. Gas et al., "Loss of OPA1 perturbates the mitochondrial inner membrane structure and integrity, leading to cytochrome c release and apoptosis," Journal of Biological Chemistry, vol. 278, no. 10, pp. 7743-7746, 2003.

[45] N. J. Van Bergen, J. G. Crowston, L. S. Kearns et al., "Mitochondrial oxidative phosphorylation compensation may preserve vision in patients with OPA1-linked autosomal dominant optic atrophy," PLoS One, vol. 6, no. 6, article e21347, 2011.

[46] C. Zanna, A. Ghelli, A. M. Porcelli et al., "OPA1 mutations associated with dominant optic atrophy impair oxidative phosphorylation and mitochondrial fusion," Brain, vol. 131, no. 2, pp. 352-367, 2008.

[47] N. Matsuda, S. Sato, K. Shiba et al., "PINK1 stabilized by mitochondrial depolarization recruits Parkin to damaged mitochondria and activates latent Parkin for mitophagy," The Journal of Cell Biology, vol. 189, no. 2, pp. 211-221, 2010.

[48] N. Sallam and I. Laher, "Exercise modulates oxidative stress and inflammation in aging and cardiovascular diseases," Oxidative Medicine and Cellular Longevity, vol. 2016, Article ID 7239639, 32 pages, 2016.

[49] X. Qiu, K. Brown, M. D. Hirschey, E. Verdin, and D. Chen, "Calorie restriction reduces oxidative stress by SIRT3- mediated SOD2 activation," Cell Metabolism, vol. 12, no. 6, pp. 662-667, 2010.

[50] G. A. Porter, W. R. Urciuoli, P. S. Brookes, and S. M. Nadtochiy, "SIRT3 deficiency exacerbates ischemia-reperfusion injury: implication for aged hearts," American Journal of PhysiologyHeart and Circulatory Physiology, vol. 306, no. 12, pp. H1602-H1609, 2014.

[51] S. Karvinen, M. Silvennoinen, P. Vainio et al., "Effects of intrinsic aerobic capacity, aging and voluntary running on skeletal muscle sirtuins and heat shock proteins," Experimental Gerontology, vol. 79, pp. 46-54, 2016.

[52] D. Geenen, P. Buttrick, and J. Scheuer, "Cardiovascular and hormonal responses to swimming and running in the rat," Journal of Applied Physiology, vol. 65, no. 1, pp. 116-123, 1988.

[53] J. C. Brown, M. R. Rickels, A. B. Troxel et al., "Dose-response effects of exercise on insulin among colon cancer survivors," Endocrine-Related Cancer, vol. 25, no. 1, pp. 11-19, 2018.

[54] O. Ronsen, E. Haug, B. K. Pedersen, and R. Bahr, "Increased neuroendocrine response to a repeated bout of endurance exercise," Medicine and Science in Sports and Exercise, vol. 33, no. 4, pp. 568-575, 2001.

[55] E. Børsheim and R. Bahr, "Effect of exercise intensity, duration and mode on post-exercise oxygen consumption," Sports Medicine, vol. 33, no. 14, pp. 1037-1060, 2003. 


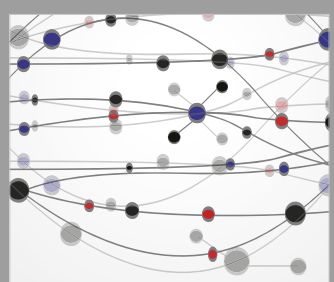

The Scientific World Journal
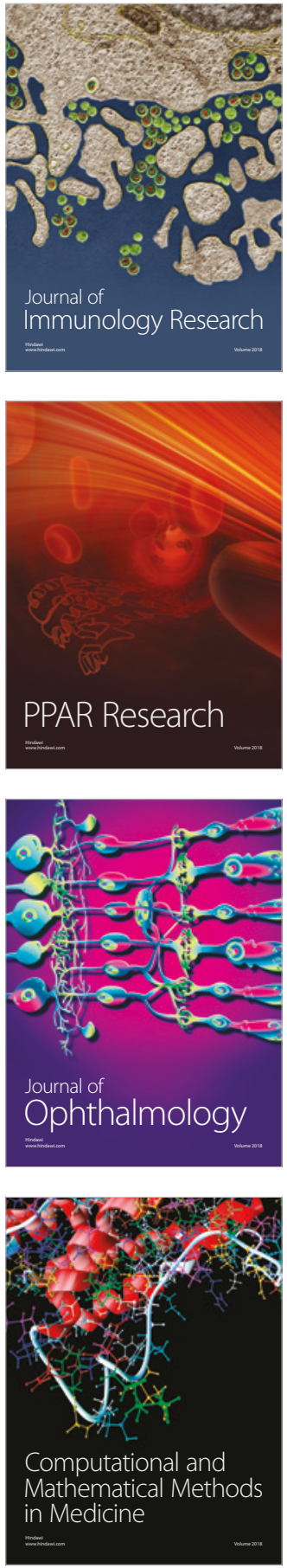

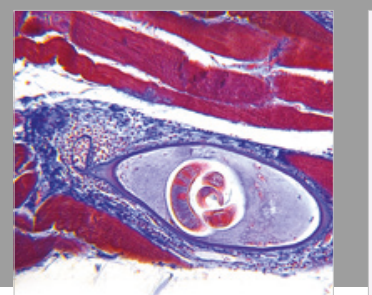

Gastroenterology Research and Practice

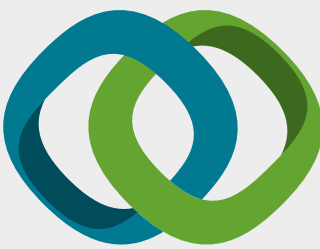

\section{Hindawi}

Submit your manuscripts at

www.hindawi.com
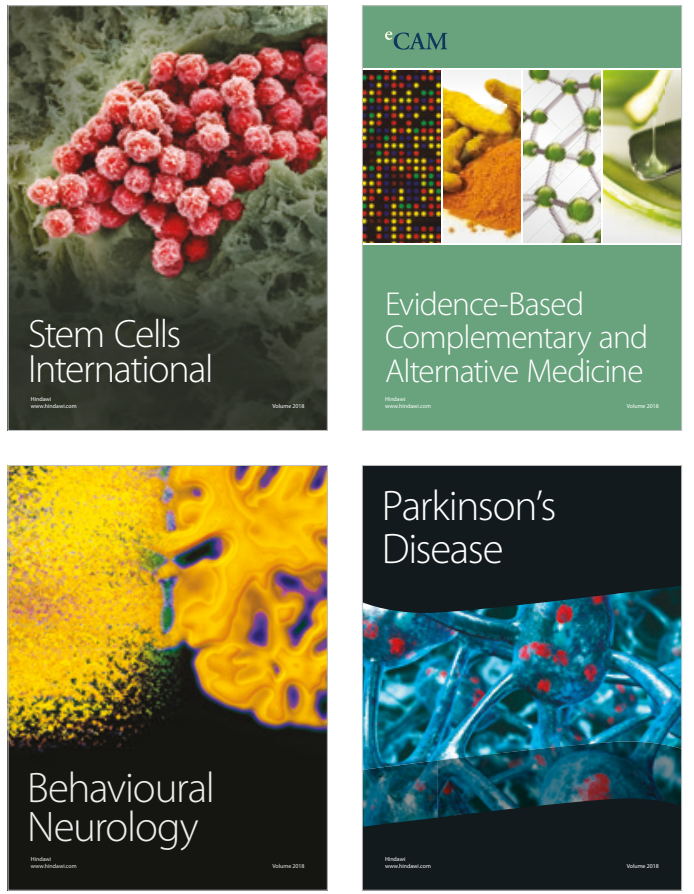

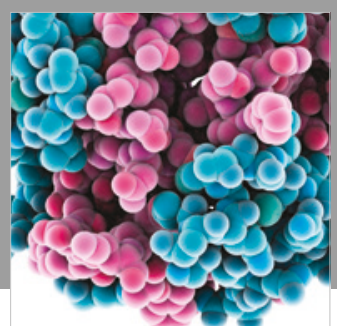

ournal of

Diabetes Research

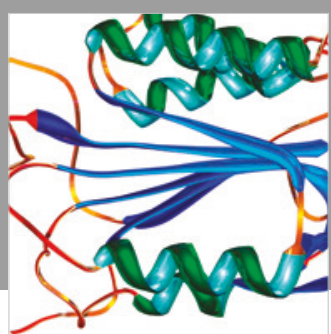

Disease Markers
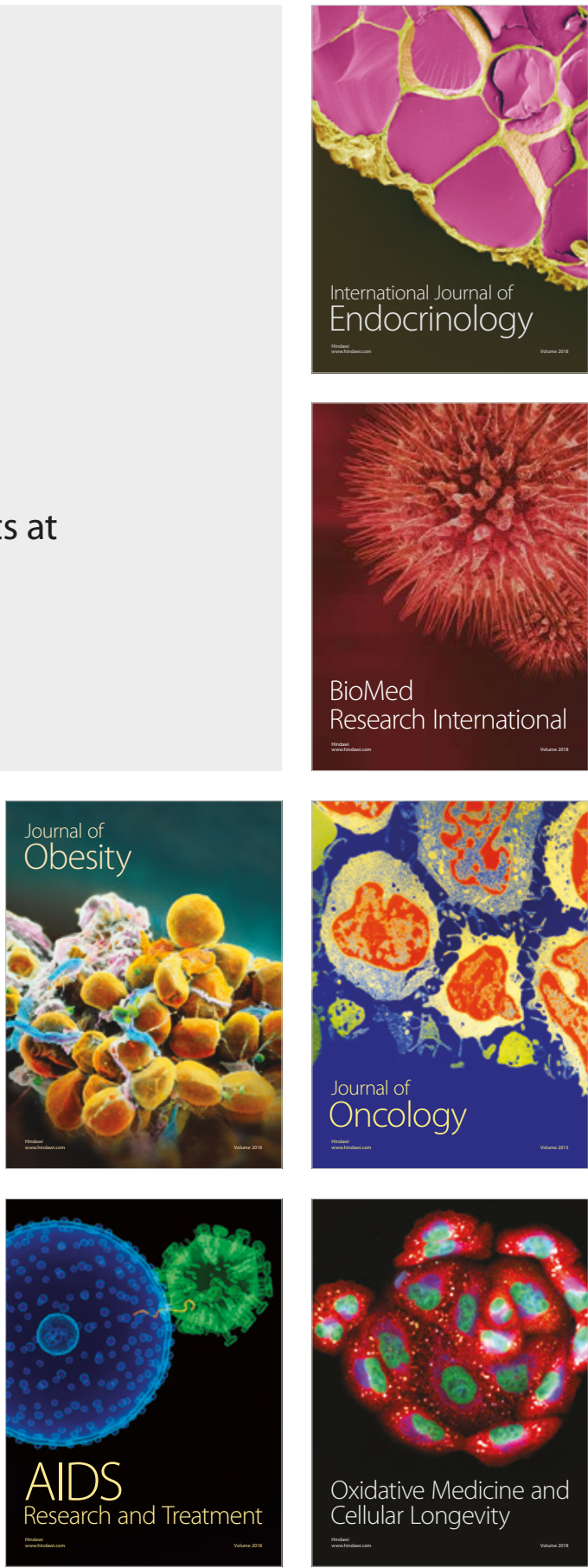